\title{
Flow structure and optical beam propagation in high-Reynolds-number gas-phase shear layers and jets
}

\author{
By P. E. DIMOTAKIS, H. J. CATRAKIS $\dagger$ \\ AND D. C. FOURGUETTE \\ Graduate Aeronautical Laboratories, California Institute of Technology, Pasadena, CA 91125, USA
}

(Received 6 May 1999 and in revised form 16 October 2000)

We report on the structure of the scalar index-of-refraction field generated by turbulent, gas-phase, incompressible and compressible shear layers and incompressible jets, and on associated beam-propagation aero-optical phenomena. Using simultaneous imaging of the optical-beam distortion and the turbulent-flow index-ofrefraction field, wavefront-phase functions were computed for optical beams emerging from the turbulent region in these free-shear flows, in an aero-optical regime producing weak wavefront distortions. Spatial wavefront-phase behaviour is found to be dominated by the large-scale structure of these flows. A simple level-set representation of the index-of-refraction field in high-Reynolds-number, incompressible shear layers is found to provide a good representation of observed wavefront-phase behaviour, indicating that the structure of the unsteady outer boundaries of the turbulent region provides the dominant contributions.

\section{Introduction}

Aero-optical phenomena involve the propagation of transmitted beams, such as lasers, and imaging beams, and are encountered in ground-based astronomical and other observations, Earth observations from rapidly moving airborne platforms and, to a lesser extent, from Space. In the course of this propagation, wavefront coherence of the transmitted/received optical beam is distorted by index-of-refraction fluctuations in the intervening turbulent medium, often compromising its utility. The discussion in this paper addresses issues for which aero-optical interactions can be accounted for in terms of wavefront-phase descriptions.

In aero-optical interactions between a propagating optical beam and a turbulent region, the resulting wavefront-phase degradation may loosely be regarded as the consequence of two propagation regimes. In propagation through the atmosphere, for example, small-amplitude, relatively long-time-scale distortions are produced, accumulated over long propagation distances (e.g. Chernov 1960; Tatarskii 1961; Kelsall 1973; Clifford 1978; Roddier 1981; Gilbert 1982a; Goodman 1985; and Tyson 1991). A different regime is encountered when propagating through a relatively confined turbulent region, as occurs when imaging through a window and its adjacent boundary layer on a fast-moving, airborne platform, for example (e.g. Liepmann 1952; Sutton 1969, 1985; Gilbert 1982b; Kelsall 1982; Klein et al. 1990; Havener 1992; Jumper

$\dagger$ Present address: Mech. \& Aerospace Eng., EG4218, U.C. Irvine, Irvine, CA 92697-3975, USA. $\ddagger$ Present address: VioSense Corp., 2400 Lincoln Ave., Altadena, CA 91001, USA. 
\& Hugo 1992, 1995; Malley, Sutton \& Kincheloe 1992; Wissler \& Roshko 1992; Fourguette, Dimotakis \& Ching 1995; McMackin et al. 1995; Cicchiello \& Jumper 1997).

Potential means to address such distortions depend on the characteristics of each type, or combination, of these regimes. Long-range atmospheric wavefront-phase distortion, resulting in the scintillation (twinkling) of stars in ground-based telescopes, for example, is characterized by order-millisecond, atmospheric-turbulence time scales. Such time scales allow deformable-mirror adaptive-optics techniques in a feedbackcontrol loop for substantial real-time wavefront-phase error corrections (e.g. Babcock 1953, 1990; Tyson 1991).

Near-field effects, as would be produced in traversing typical boundary layers or shear layers, are typically characterized by time scales that are too short to permit conventional adaptive-optics techniques. By way of example, a boundary layer with a thickness of $\delta_{\mathrm{bl}} \approx 1 \mathrm{~cm}$, on the exterior of an optical window on an airplane moving at $U_{\infty} \approx 250 \mathrm{~m} \mathrm{~s}^{-1}$ (ca. 500 knots), would be characterized by a frequency spectrum scaled by a characteristic large-scale boundary-layer (outer) time of

$$
t_{\mathrm{bl}}=\frac{\delta_{\mathrm{bl}}}{U_{\infty}} \simeq 4 \times 10^{-5} \mathrm{~s} .
$$

Separated shear layers from the same airplane tend to be thicker, say, $\delta_{\mathrm{sl}} \approx 10-50 \mathrm{~cm}$, and possess longer characteristic (outer) times, i.e.

$$
t_{\mathrm{sl}}=\frac{\delta_{\mathrm{sl}}}{U_{\infty}} \simeq 4 \times 10^{-4}-2 \times 10^{-3} \mathrm{~s} .
$$

At the high Reynolds numbers relevant to these scenarios, the frequency content of index-of-refraction fluctuation spectra is very large, with fluctuation (inner) time scales extending to several orders of magnitude lower, corresponding to very high frequencies, albeit at lower amplitudes.

In these applications, progress is hampered by incomplete knowledge of the geometrical structure of the index-of-refraction field in the turbulent region through which the optical beam must propagate. Successes to date using adaptive optics, for example, rest more with the general power of closed-loop control systems, rather than good models of the system being controlled. The 'system', in this case, is the intervening turbulent medium and 'good models' would require a description of the dynamics of turbulence with some fidelity.

Weak aero-optical effects of optical-beam propagation through a confined turbulent region can be described, to a good approximation, in terms of geometrical optics and the resulting wavefront-phase function (eikonal equation-Born \& Wolf 1993),

$$
\varphi=\int \boldsymbol{k}(\boldsymbol{x}, t) \cdot \mathrm{d} \boldsymbol{x},
$$

for each beam (ray) that emerges from the turbulent-flow domain. In this expression,

$$
\boldsymbol{k}=\hat{\boldsymbol{k}} \frac{2 \pi}{\lambda}
$$

is the local wavevector of the beam, with $\lambda$ the local value of the wavelength. It is altered in direction, $\hat{\boldsymbol{k}}$, and magnitude,

$$
k=\frac{2 \pi}{\lambda}=\frac{2 \pi n}{\lambda_{0}}=k_{0} n,
$$

by the fluctuating index-of-refraction field, $n(\boldsymbol{x}, t) \equiv c_{0} / c(\boldsymbol{x}, t)=\lambda_{0} / \lambda(\boldsymbol{x}, t)$, where $k_{0}$, 
$c_{0}$, and $\lambda_{0}$ are the wavevector magnitude, speed of light, and wavelength of light in vacuum, respectively. As far as light propagation is concerned, the high speed of light, relative to all flow speeds of interest here, permits the description of aero-optical interactions as if the flow is spatially frozen, at any one time. Any time dependence of the resulting aero-optical interactions is that of the turbulent flow field.

For small deflections of the wavevector, the change in local propagation direction can be neglected, to leading order, and the integral (2) can be approximated as a wavefront-phase function, $\varphi\left(x, y ; z_{1}\right)$, in terms of coordinates in a $\left(z=z_{1}\right)$-plane perpendicular to the nominal beam-propagation direction, $z$, at any one time, $t$, i.e.

$$
\varphi\left(x, y ; z_{1}\right)=k_{0} \int^{z_{1}} n(x, y, z) \mathrm{d} z .
$$

The ratio $\varphi / k_{0}$ is sometimes referred to as the (effective) optical path length (OPL) between the source and points in the plane $\left(x, y ; z_{1}\right)$.

For gas-phase flows, as investigated here, indices of refraction are near unity. By way of example, $n_{\text {air }}-1 \simeq 2.78 \times 10^{-4}$, at $\lambda_{0}=540 \mathrm{~nm}, T=15^{\circ} \mathrm{C}$, and $p=1$ bar, with $n-1$ for helium nearly an order of magnitude smaller. If the index-of-refraction fluctuations are scaled by $\Delta n$, e.g. if they arise as the result of mixing of two fluids with indices of refraction $n_{1}$ and $n_{2}$, corresponding, for example, to the indices of refraction of two free-stream fluids on either side of an incompressible turbulent shear-layer region, then the maximum index-of-refraction excursion will be given by

$$
\Delta n=\left|n_{1}-n_{2}\right| .
$$

If the variation in $n(\boldsymbol{x})$ is not too large, the leading-order beam-propagation effect is the phase accumulation in the original beam-propagation direction, $z$, as a function of the coordinates, $(x, y)$, in the propagation-normal plane at $z_{1}(2 d)$. This will be scaled by the index-of-refraction magnitude, $\Delta n$, and the extent of the turbulent region, $L$, in the direction of propagation, $z$. This motivates the definition of a scaled propagation perturbation phase function (Chow 1975; Steinmetz 1982),

$$
\tilde{\varphi}\left(x, y ; z_{1}\right) \equiv \frac{\varphi\left(x, y ; z_{1}\right)}{k_{0} L \Delta n} \simeq \frac{1}{L \Delta n} \int^{z_{1}}\left[n(x, y, z)-n_{\infty}\right] \mathrm{d} z,
$$

which can be used to characterize weak aero-optical effects, in the geometrical-optics limit, stemming from confined, turbulent-flow index-of-refraction regions. In the case of a binary mixture, for example, for which the local index of refraction is the result of the local mixture composition, we have (for uniform number density)

$$
\tilde{\varphi}\left(\xi, \eta ; \zeta_{1}\right) \simeq \int^{\zeta_{1}} X(\xi, \eta, \zeta) \mathrm{d} \zeta,
$$

where

$$
X(\xi, \eta, \zeta)=\frac{n(\xi, \eta, \zeta)-n_{1}}{n_{2}-n_{1}}
$$

is the mole fraction (conserved scalar) of, say, the high-index-of-refraction species in the binary mixture, and $(\xi, \eta, \zeta)=(x / L, y / L, z / L)$. This expression will be used to illustrate some of the aero-optical effects in beam propagation through turbulent shear layers and jets that mix species with different indices of refraction (different Dale-Gladstone constants).

Wavefront-phase functions from turbulent regions are typically estimated in practice using direct-measurement techniques, such as Shack-Hartmann lenslet arrays, 
shearing interferometers, etc. Quantitative planar-imaging techniques, however, permit a direct measurement of the instantaneous index-of-refraction field in a plane, i.e. $n\left(x, y=y_{1}, z\right)$. This is sufficient to quantify the scaled wavefront-phase function along one spatial dimension, i.e. $\tilde{\varphi}\left(x, y=y_{1}, z=z_{1}\right)$ in (4), corresponding to the propagation of an optical sheet in the $\left(y=y_{1}\right)$-plane, allowing a numerical computation of the scaled wavefront-phase propagation function. The resulting $\tilde{\varphi}\left(x, y_{1}, z_{1}\right)$ scaled wavefront-phase functions will be used as a measure of the aero-optical interactions in high-Reynolds-number shear layers and jets in the discussion below.

\section{Experimental method}

The measurements in shear layers and jets described below relied on Rayleigh scattering to infer the instantaneous index-of-refraction field $(2 d), n\left(x, z ; y=y_{1}, t=t_{1}\right)$, in the $\left(y=y_{1}\right)$-plane illuminated by a thin laser sheet. The index-of-refraction field was generated, in turn, by the turbulent mixing of two gases with different indices of refraction, $n_{1}$ and $n_{2}$, respectively.

Rayleigh scattering is not widely used as a gas-phase imaging diagnostic. Its main difficulty stems from the fact that it is elastic. Stray light from reflections/refractions from windows, other solid boundaries, or dust and aerosols is at the same frequency/wavelength and cannot be easily differentiated. Such scattering can overwhelm the weak scattering from gas molecules. These difficulties can be overcome, however, by avoiding glass windows anywhere near the field of view, minimizing stray light incident in the collection optics, and taking care to run the experiments in enclosed facilities operated in as nearly particle-/aerosol-free environments as is feasible. Nevertheless, a few, small, high-intensity spots from dust and liquid $\mathrm{C}_{2} \mathrm{H}_{4}$ and possibly other aerosols in the raw images can occasionally be detected. In these experiments, they were removed interactively in a post-processing step, using either bilinear interpolation in the small excised areas (shear-layer data), or two-dimensional Coons (1967) patches (for the jet data).

Experiments on both flows, shear layers and jets, relied on scattering from the beam of a frequency-doubled Nd:YAG $(\lambda=532 \mathrm{~nm})$, pulsed $(\tau \simeq 9 \mathrm{~ns})$ laser (Lumonics YM-1000), with an energy $E_{\mathrm{p}} \simeq 300 \mathrm{~mJ} /$ pulse and a pulse-repetition frequency of $10 \mathrm{~Hz}$. The beam was expanded in the streamwise direction, using cylindrical optics, focused in the plane of the laser sheet using long-focal-length spherical optics, and steered into the test section through an optical window, with adequate subtended solid-angle constraints, some distance from the field of view, to limit stray-light contamination.

The Rayleigh-scattered light was collected with the optical-collection axis at right angles and imaged through one of the test-section optical windows with a $35 \mathrm{~mm}$ camera lens (Minolta, $85 \mathrm{~mm}$ focal length, F/1.9). Rayleigh-scattering image data were recorded on cryogenically cooled, $(1024 \times 1024)$-pixel, CCD cameras. Shearlayer images were recorded on a Photometrics CCD Series-200 camera. Jet images were recorded on a (lower-noise, higher quantum-efficiency) Princeton Instruments CCD camera (Model TKB1024-1; thinned, back-illuminated CCD). A suitable time delay was introduced between flow onset and image acquisition to allow steady-state conditions to be established before each image was recorded. The externally controlled camera shutter was opened to admit a single laser pulse only. Images were digitized at 16 bits/pixel. The long time required for a high signal-to-noise frame readout from these cameras $\left(t_{\text {readout }} \simeq 20 \mathrm{~s}\right)$ permitted only one image to be recorded from each run, in both the shear-layer and jet short-running-time experiments. 
The measurement method relies on the different index of refraction and, hence, different Rayleigh-scattering cross-section, of the species comprising the binary mixture. The complex index-of-refraction field is the result of the variable mixture composition in the turbulent mixing zone. This may be expressed in terms of the refractive index of the gas mixture, $n$, and the number density of scatterers in the probe volume, $N_{\mathrm{sc}}$ (e.g. Penney 1969),

$$
\frac{\partial \sigma}{\partial \Omega}=\frac{\pi^{2}}{N_{\mathrm{sc}}^{2} \lambda^{4}}\left(n^{2}-1\right)^{2}\left(1+\cos ^{2} \theta_{\mathrm{sc}}\right)
$$

where here, $\lambda=532 \mathrm{~nm}$ is the wavelength of the (doubled-Nd:YAG) laser light, and $\theta_{\text {sc }}$ is the (scattering) angle between the incident light and the direction of light collection. For an index of refraction close to unity, i.e.

$$
n(\boldsymbol{x})=1+n^{\prime}(\boldsymbol{x}), \quad \text { with } n^{\prime} \propto N_{\mathrm{sc}}, \quad \text { and } \quad n^{\prime} \ll 1,
$$

the differential scattering cross-section is effectively independent of the scatterer number density, $N_{\mathrm{sc}}$. For an ideal-gas mixture, the differential Rayleigh-scattering cross-section is well-approximated as a mole-fraction-weighted sum of the individual species differential-scattering cross-sections, i.e.

$$
\frac{\partial \sigma_{\mathrm{mix}}}{\partial \Omega}=\sum_{i} X_{i} \frac{\partial \sigma_{i}}{\partial \Omega}
$$

The intensity of the Rayleigh-scattered light, $I_{\mathrm{R}}$, collected from a laser beam of length $\ell_{\mathrm{c}}$, in the direction of the solid angle, $\Omega$ (here at $90^{\circ}$ ), is given by

$$
I_{\mathrm{R}} \propto I_{0} N_{\mathrm{sc}} \frac{\partial \sigma_{\mathrm{mix}}}{\partial \Omega} \Delta \Omega \ell_{\mathrm{c}} \eta_{\mathrm{c}},
$$

where $I_{0}$ is the incident-light intensity, $\Delta \Omega$ is the solid angle of the collecting optical system, and $\eta_{\mathrm{c}}$ is the cumulative collection-optics and image-detection system efficiency. For a beam formed into a sheet and imaged onto a CCD focal-plane pixel array, as in this experiment, the local imaged intensity will be given by

$$
I_{\mathrm{R}}=\Phi_{0} N_{\mathrm{sc}} \frac{\partial \sigma_{\mathrm{mix}}}{\partial \Omega} \Delta \Omega A_{\mathrm{c}} \eta_{\mathrm{c}}
$$

where $\Phi_{0}$ is the local illuminating flux (units of $\Phi_{0}$ are units of intensity, $I$, per unit transverse laser-sheet width).

In these experiments, ethylene $\left(\mathrm{C}_{2} \mathrm{H}_{4}\right)$ was used as the high-index-of-refraction gas, with nitrogen or helium as the low-index-of-refraction gas. Nitrogen was used as the low-index-of-refraction gas in all jet flows, as well as in the low- and moderateMach-number shear-layer flows. At the same pressure and temperature, $\mathrm{C}_{2} \mathrm{H}_{4}$ and $\mathrm{N}_{2}$ are density-matched (same molecular mass of 28, barring isotopic-composition variations), whereas, $\rho_{\mathrm{C}_{2} \mathrm{H}_{4}} / \rho_{\mathrm{He}}=7$ (at the same $p$ and $T$ ).

For a binary mixture, the differential Rayleigh-scattering cross-section can be expressed in terms of the mole fraction of either of the two scattering species, i.e.

$$
\begin{aligned}
\frac{\partial \sigma_{\mathrm{mix}}}{\partial \Omega}(x, z) & =[1-X(x, z)] \frac{\partial \sigma_{1}}{\partial \Omega}+X(x, z) \frac{\partial \sigma_{2}}{\partial \Omega} \\
& =\left[\left(1-\frac{1}{\alpha}\right) X(x, z)+\frac{1}{\alpha}\right] \frac{\partial \sigma_{2}}{\partial \Omega},
\end{aligned}
$$

where $X(x, z)$ is the high- $\sigma$-species mole fraction. For the binary mixture used here, 
we have

$$
\frac{1}{\alpha} \equiv \frac{\sigma_{1}}{\sigma_{2}} \simeq \begin{cases}1 / 5.7 & \text { for } \mathrm{N}_{2} / \mathrm{C}_{2} \mathrm{H}_{4} \\ 1 / 234 & \text { for } \mathrm{He} / \mathrm{C}_{2} \mathrm{H}_{4} .\end{cases}
$$

In incompressible, isothermal, ideal-gas flows, $N_{\mathrm{sc}}$ may be treated as spatially uniform. The local scattered intensity then measures the local mole fraction of, say, $\mathrm{C}_{2} \mathrm{H}_{4}$ in the binary mixture, e.g. (10) and Dyer (1979). For indices of refraction close to unity (6), the Rayleigh-scattered intensity is a linear function of the composition mole fraction (10), i.e.

$$
I(x, z) \propto c_{1} X+c_{0} .
$$

In compressible flow, however, $I_{\mathrm{R}}(x, z)$ registers the combined effects of the nonuniform mole-fraction and number-density fields. For an ideal gas

$$
N_{\mathrm{sc}}(x, z) \propto \frac{p(x, z)}{T(x, z)},
$$

and therefore

$$
I_{\mathrm{R}}(x, z) \propto \Phi_{0}(x, z) \frac{p(x, z)}{T(x, z)}\left[\left(1-\frac{1}{\alpha}\right) X(x, z)+\frac{1}{\alpha}\right] .
$$

The imaged intensity can be seen to be a function of the local laser illumination flux, the pressure and temperature (i.e. local number density), as well as fluid composition.

The detected light-intensity signal, $I_{\mathrm{d}}(x, z)$ is the sum of three contributions,

$$
I_{\mathrm{d}}(x, z)=I_{\mathrm{R}}(x, z)+I_{\mathrm{b}}(x, z)+I_{\mathrm{n}}(x, z),
$$

where $I_{\mathrm{R}}(x, z)$ is the Rayleigh-scattered light from the mixture in the probe volume (12), $I_{\mathrm{b}}(x, z)$ is the background light scattered by the flow apparatus, and $I_{\mathrm{n}}(x, z)$ is the total noise (shot + dark + readout + etc.).

Even if minimized through the use of aerodynamic windows, some residual, background-scattered light, $I_{\mathrm{b}}(x, z)$, in the image, produced by the inevitable reflections off the guidewalls and windows was still present. It was measured by acquiring a reference set of background images, $I_{\mathrm{ref}}(x, z)$, while either flowing helium (negligible Rayleigh-scattering cross-section) through the test section (top and bottom streams) in the case of the shear-layer experiment, or imaging an evacuated vessel, in the case of the jet experiments. The gas-phase scattering data were calculated by subtracting reference background-noise data,

$$
I_{\mathrm{ref}}(x, z)=\left[I_{\mathrm{R}}(x, z)\right]_{\mathrm{He}}+I_{\mathrm{b}}(x, z)+I_{\mathrm{n}}(x, z) \simeq I_{\mathrm{b}}(x, z)+I_{\mathrm{n}}(x, z) .
$$

The Rayleigh-scattering signal from the gas mixture was estimated, in turn, by subtracting the ensemble-averaged reference background images, $I_{\text {ref }}(x, z)$, i.e.

$$
I_{\mathrm{R}}(x, z) \simeq I_{\mathrm{d}}(x, z)-\left\langle I_{\mathrm{ref}}(x, z)\right\rangle .
$$

There are two major sources of spatial non-uniformity in $\Phi_{0}(x, z)$, the laser-sheet illumination (9). The first is caused by the (truncated) near-Gaussian beam (streamwise) profile and by the in-plane geometrical divergence of the laser sheet that emanates from a virtual origin some distance from the test section. The second is the result of aero-optic effects introduced by the inhomogeneous index-of-refraction field in the mixing-layer region, through which the illuminating laser sheet propagates, as discussed in the Introduction. The latter effects are, of course, a focus of this study.

While the non-uniformity of the laser beam profile is (almost) the same for each 
laser pulse and can be removed by normalizing the images accordingly (flat-fielding), the aero-optical distortion caused by the turbulent region itself is unique to each realization and identifiable as a high-spatial-frequency optical pattern (radial streaks) in the laser-sheet intensity, $\Phi_{0}(x, z)$. In addition, there are residual shot-to-shot variations in laser-sheet illumination profile that exceed the nascent signal-to-noise limitations in these measurements and must be corrected for if the accuracy potential of these measurements is to be realized. The methods for dealing with these in each of the two flow geometries were different and will be described below.

For the Rayleigh-scattering measurements of the two flows described in this paper, imaging uniform-composition fluid composed of the high- $\sigma$ gas (i.e. $\mathrm{C}_{2} \mathrm{H}_{4}, X(x, z)=1$ ) over some region, yielded signal-to-noise ratios dominated by photon shot noise. This results in a fractional root-mean-square (r.m.s.) deviation of local detected values from their mean that is close to the quantum limit, i.e. the reciprocal of the square-root of the number of detected photons (CCD electrons) per pixel (examples of actual numbers will be given below), in contrast to flow imaging that relies on smoke or other Mie-scattering fluid markers. The latter is usually dominated by marker shot noise, leading to fractional r.m.s. deviations given by the (typically, much larger) reciprocal of the square root of the number of markers (scattering particles) imaged per pixel.

\section{Beam propagation through turbulent shear layers}

The shear-layer investigations were conducted in the GALCIT Supersonic Shear Layer $\left(\mathrm{S}^{3} \mathrm{~L}\right)$ facility (Hall 1991; Slessor 1998; Slessor, Bond \& Dimotakis 1998). They are based on data recorded in a previous investigation (Fourguette, Bond \& Dimotakis 1993) and processed anew, as described here, for the aero-optical study of wavefront-phase behaviour through shear layers. The brief description of the shear-layer flow facility and experiments below is included for completeness.

\subsection{Experiments}

The $\mathrm{S}^{3} \mathrm{~L}$ facility is a two-stream, blow-down wind tunnel, nominally operating with an atmospheric test-section (static) pressure and a run time of $t_{\text {run }} \approx 2-3 \mathrm{~s}$, for highMach-number flows, or slightly longer for subsonic and low-Mach-number flows. Gas for each of the two free streams is supplied by independent flow systems. The nominally high-speed (subsonic or supersonic) stream is generated from a pressurecontrolled plenum section by a converging or converging-diverging nozzle, as required to produce the desired flow speed and Mach number. A schematic of the test section, with nozzle blocks for subsonic flow in both free streams, is shown in figure 1.

A schematic of the optical diagnostic set-up is depicted in figure 2. The laser platform is mounted on the test-section support frame to minimize beam deflection caused by vibrations and relative displacements during the run. Optical glass windows in the flow test section were avoided to minimize scattered background light from solid surfaces near the field of view. Instead, the laser sheet was passed through aerodynamic windows (Parmentier \& Greenberg 1973) composed of narrow (ca. $2 \mathrm{~mm}$ ) streamwise slits, at midspan in the top and bottom guidewalls (Rosemann, Dimotakis \& Hall 1992).

In these experiments, the centre of the imaged region is $22 \mathrm{~cm}$ downstream from the splitter-plate trailing edge. A CCD pixel images a volume, $V_{\text {meas }} \simeq 105 \times 105 \times \ell_{y} \mu \mathrm{m}^{3}$ in the flow, where $\ell_{y}$ is the laser-sheet thickness (in the spanwise direction). The lasersheet thickness at the waist was estimated to be $\ell_{y 0} \approx 300 \mu \mathrm{m}$. The sheet was aligned 


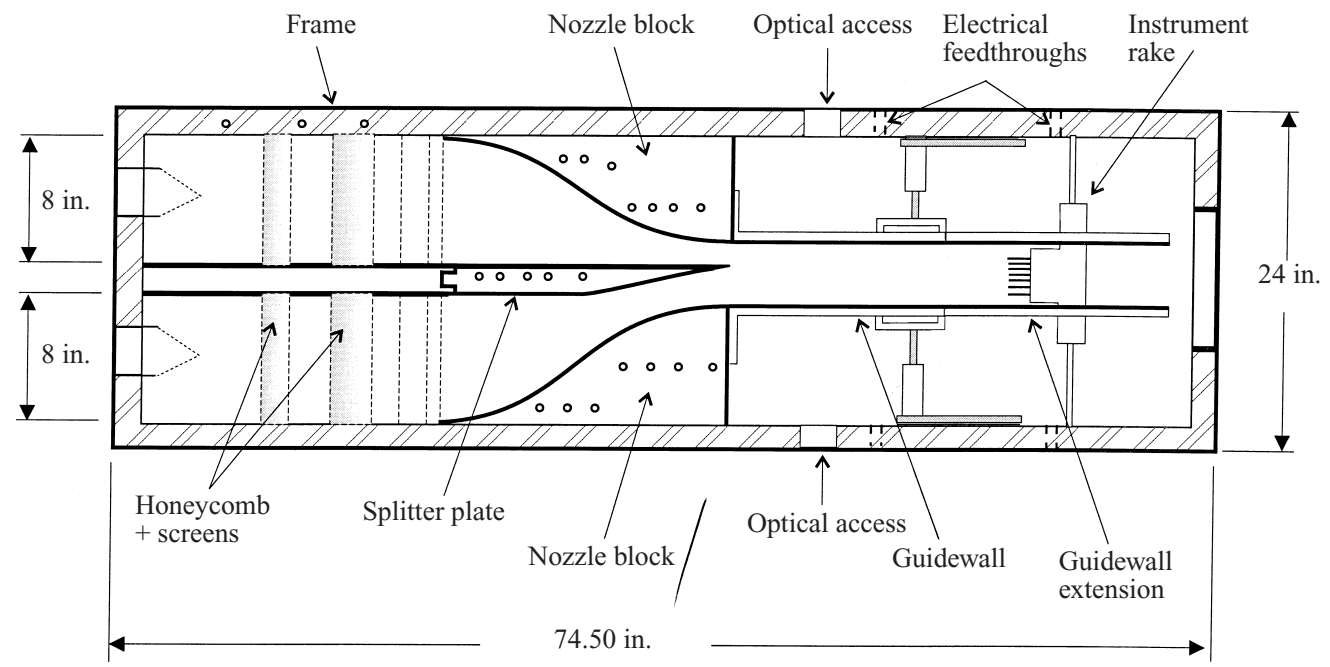

FIGURE 1. Shear-layer flow test-section schematic, configured for subsonic free streams.

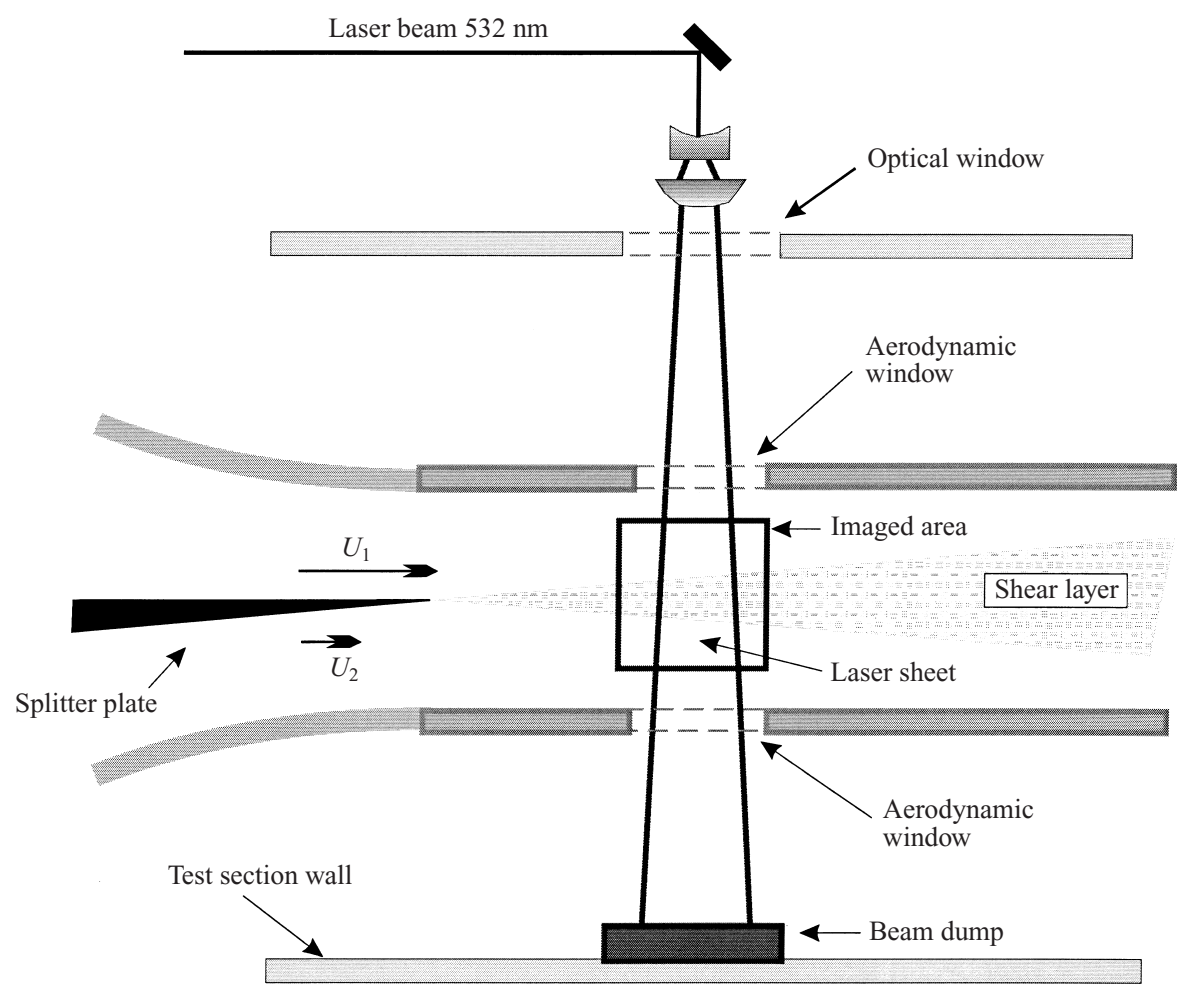

FIGURE 2. Schematic of optical configuration for shear-layer data.

through the aerodynamic-window pair, along the midspan plane, with a spanwise waist positioned at the shear-layer flow mixing-zone mid-height. The vertical extent of the imaged region was well within the Rayleigh range of the laser sheet, estimated to be within $\pm 7 \mathrm{~cm}$ from the test section midheight.

Experiments were conducted at three flow compressibilities, corresponding to total 


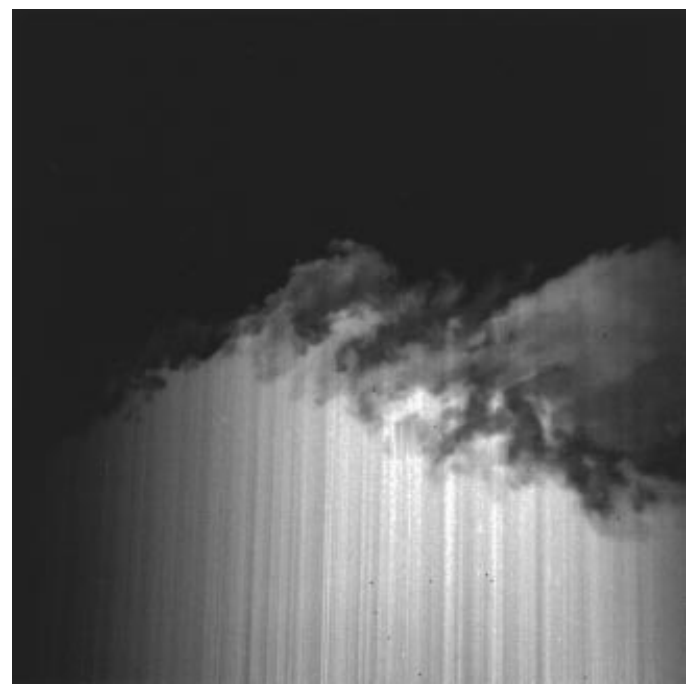

FIGURE 3. Rayleigh-scattering scalar-field image for low-compressibility shear-layer flow (subsonic, $M_{\mathrm{c}} \simeq 0.15$ ) realization, uncorrected for laser-beam non-uniformity and aero-optic effects. Grey-scale level denotes scattering intensity.

convective Mach numbers (Papamoschou 1989)

$$
M_{\mathrm{c}} \equiv \frac{U_{1}-U_{2}}{a_{1}+a_{2}} \simeq 0.15,0.54, \text { and } 0.96,
$$

referred to, here, as the low-, moderate-, and high-compressibility cases, respectively.

The high-compressibility case was tailored to exploit the high speed of sound as well as the negligible Rayleigh-scattering cross-section (cf. 10b) of helium, which was used as the high-speed gas, relative to $\mathrm{C}_{2} \mathrm{H}_{4}$. As a consequence, for the high-compressibility flow, the imaged scattering signal effectively measures the local number density of low-speed free-stream fluid $\left(\mathrm{C}_{2} \mathrm{H}_{4}\right)$, only.

The resulting optical pattern for a high-Reynolds-number $\left(\operatorname{Re}_{\delta} \simeq 2 \times 10^{5}\right)$, lowcompressibility (subsonic, $U_{2} / U_{1} \simeq 0.5, \rho_{2} / \rho_{1} \simeq 1, M_{\mathrm{c}} \simeq 0.15$ ) shear-layer realization is illustrated in figure 3 . Flow in all cases is from left to right, with the laser sheet propagating from top to bottom to minimize aero-optical effects across the input (top) aerodynamic window (same gas across that interface). The high-speed free stream occupies the upper part and the low-speed free stream the lower part of the imaged regions. Aero-optical distortions, manifested as streaks in the high-index-ofrefraction free stream fluid (bottom), are most conspicuous in flows of mixtures of species with large differences in index of refraction, with sharp composition gradients, as in these experiments. Optical deflections in the imaged $(x, z)$-plane are registered directly as changes in the direction of propagation of individual rays. Their most conspicuous consequence in these data, however, is the resulting variation in intensity in the otherwise uniform scattering medium (pure $\mathrm{C}_{2} \mathrm{H}_{4}$, in this case). Specifically, converging ray bundles generate higher local intensity, whereas diverging ray bundles generate lower local intensity, leading to the streaky appearance in the bottom half of the images.

Out-of-plane deflections, as would arise from components of index-of-refraction gradients normal to the $(x, z)$-plane, do not lead to intensity variations, provided the resulting rays remain within the depth of field of the receiving optical system, 
as was the case for all experiments reported here. This allows the measurement to register the in-plane, $\partial n / \partial x$ (streamwise) and $\partial n / \partial z$ (cross-stream) index-of-refraction gradient components, but to remain essentially insensitive to out-of-plane, $\partial n / \partial y$, gradient components.

Following background subtraction (15), the images are first corrected for the aerooptical streak non-uniformities using a variation on the image-processing method employed by Rosemann et al. (1992). In particular, the (x,z)-coordinate system of the images is mapped onto a polar coordinate system, $(r, \theta)$, whose origin matches the virtual origin of the diverging laser sheet. $\dagger$ Values of the imaged Rayleighscattered intensity, $I_{\mathrm{R}}(x, z)$, are mapped to obtain $I_{\mathrm{R}}(r, \theta)$, using a continuous (subpixel) interpolation scheme. For a fixed polar angle, $\theta$, in the plane of the laser sheet, the illumination field, $\Phi_{0}(r, \theta)$, may be regarded as (nearly) constant along rays. Along a constant $-\theta$ ray, the scattered-light intensity, $I_{\mathrm{R}}(r, \theta)$, is normalized by the difference in free-stream intensity values along that ray, i.e.

$$
I(r, \theta)=\frac{I_{\mathrm{R}}(r, \theta)-I_{1}(\theta)}{I_{2}(\theta)-I_{1}(\theta)}
$$

where $I_{1}(\theta)$ and $I_{2}(\theta)$ are the scattered-light intensities (along the ray $\theta$ ) in the highspeed (top) and low-speed (bottom) free stream, respectively. An inverse mapping to Cartesian coordinates then yields the scalar (Rayleigh-scattering) field, $I(x, z)$, corrected for laser illumination non-uniformities as well as aero-optical streaks.

\subsection{Scalar-field structure}

The corrected Rayleigh-scattering scalar-field image is reproduced in figure $4(b)$. By way of reference, a side-view schlieren image (horizontal knife edge) from a different run at similar flow conditions, is also depicted in figure 4 ( $a$, taken from Hall 1991, figure 4.30, left), for subsonic free streams, $\mathrm{N}_{2} /[1 / 3 \mathrm{He}+2 / 3 \mathrm{Ar}], \rho_{2} / \rho_{1} \simeq 1$, $\left.M_{\mathrm{c}} \simeq 0.15\right)$. Also indicated in $(a)$ is the approximate extent and location of the field of view in the Rayleigh-scattering images, relative to the shear-layer origin. This was kept fixed in all shear-layer experiments. Schlieren images record the cumulative, spanwiseintegrated wavefront-phase distortions of the optical beam caused by spatial indexof-refraction gradients, as a function of the streamwise and cross-stream coordinates. In contrast, Rayleigh-scattering offers a planar image that registers the index-ofrefraction field in the laser-sheet-illuminated (midspan) plane.

The image-correction scheme employed can locally overcorrect in the interior of the mixed-fluid region. This is because removing the low-index-of-refraction region amplitude from the data, i.e. subtracting $I_{1}(\theta)$ in (17) along each ray, drops the (corrected) image amplitude to zero in the pure, high-speed free-stream fluid region, so no correction is applied there, with residual (scaled) errors stemming from the correction scheme confined to the mixed-fluid region. As can be seen, the scheme removes the leading-order aero-optical effects. Corrected Rayleigh-scattering shearlayer-flow images discussed below were processed by this method.

Figure 5(a) depicts a schlieren image recorded for medium-compressibility $\left(M_{1} \simeq 1.5\left[\mathrm{~N}_{2}\right], M_{2} \simeq 0.3\left[\mathrm{C}_{2} \mathrm{H}_{4}\right], U_{2} / U_{1} \simeq 0.23, \rho_{2} / \rho_{1} \simeq 0.68, M_{\mathrm{c}} \simeq 0.54\right)$ shear-layer flow conditions and figure 5(b) a (corrected) midspan-plane Rayleigh-scattering image at the same flow conditions. Evident in the schlieren image are Mach waves in the $M_{1} \simeq 1.5$ high-speed free stream (the approximate extent of the Rayleigh-scatteringimage field of view also indicated). Also visible is a distinct pair of slightly stronger

$\dagger$ In accord with the notation above, $z$ here corresponds to the beam-propagation (cross-stream) direction, usually denoted by $y$ in shear-layer flows. 
(a)

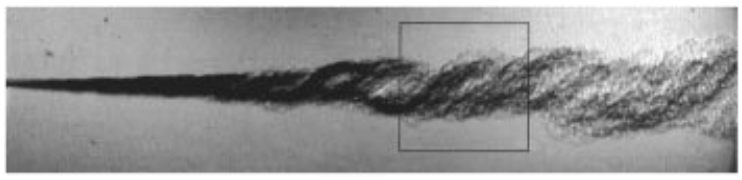

(b)

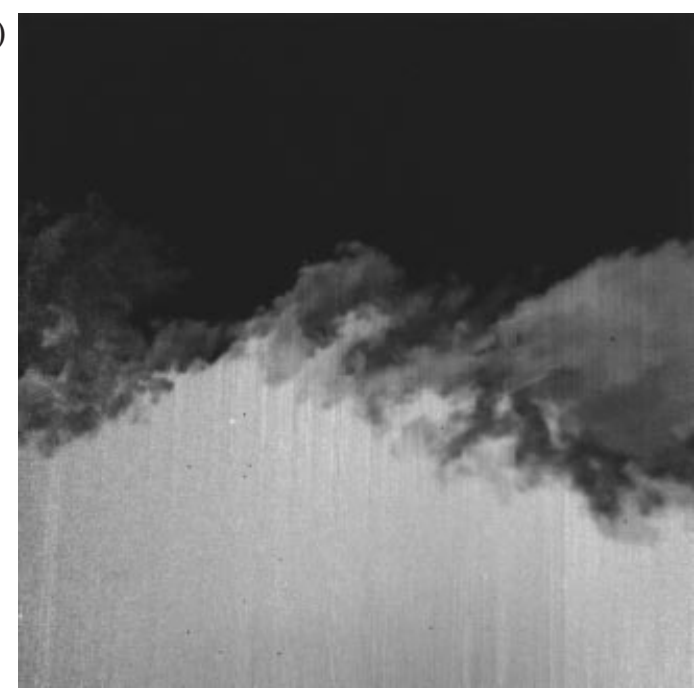

FIGURE 4. Low-compressibility $\left(M_{\mathrm{c}} \simeq 0.15\right)$ shear-layer flow. (a) Schlieren image from Hall (1991, figure 4.30, left). (b) Rayleigh-scattering image (data from figure 3), corrected for aero-optic effects. Note mixed-fluid regions of near-uniform composition (index of refraction). In this and subsequent figures, the square in $(a)$ indicates the approximate extent of Rayleigh-scattering-image field of view.

waves emanating from the downstream end of the aerodynamic window. No flow disturbances from the aerodynamic window are discernible in the boundary layer growing on the high-speed stream (top) guidewall. The image also records the bottom guidewall convergence angle, required to supply the shear-layer entrainment requirements (negative displacement thickness) for a zero streamwise pressure gradient in the test section.

A comparison of the schlieren data confirms the decrease in spanwise coherence, at large scales, as flow compressibility increases, in accord with linear-stability and numerical-simulation analyses that predict greater growth rates for oblique waves for supersonic flow (e.g. Sandham \& Reynolds 1991), and as has been noted previously experimentally (e.g. Fourguette, Mungal \& Dibble 1990; Clemens \& Mungal 1992, 1995; Hall, Dimotakis \& Rosemann 1993; Elliott, Samimy \& Arnette 1995). The spanwise-integrating schlieren visualization requires that the large-scale-structure cores are aligned with the spanwise coordinate to be registered as such. In contrast, planar Rayleigh-scattering image slices do not rely on spanwise coherence to register large-scale flow organization. The latter indicate that organized large-scale structure motion persists to this level of compressibility, as evidenced by the sharp boundaries of the scalar field, even though the cross-stream coherence of a large-scale vortical structure is not as clear as in the less-compressible flow. Sharp boundaries in the scalar field can also be seen in Rayleigh-scattering images of a supersonic shear layer in a pressure-matched jet, and the (subsonic) shear layer formed downstream of the Mach disk in an underexpanded jet (Yip et al. 1989). It is also evident that 
(a)

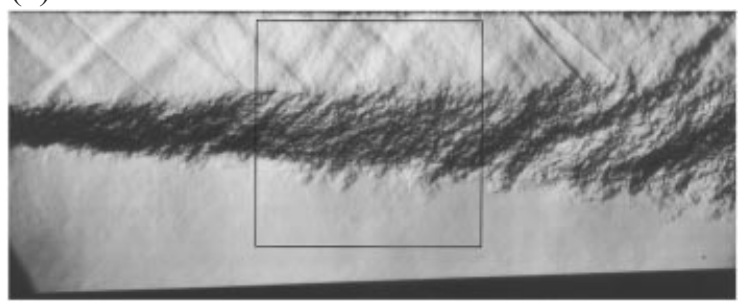

(b)

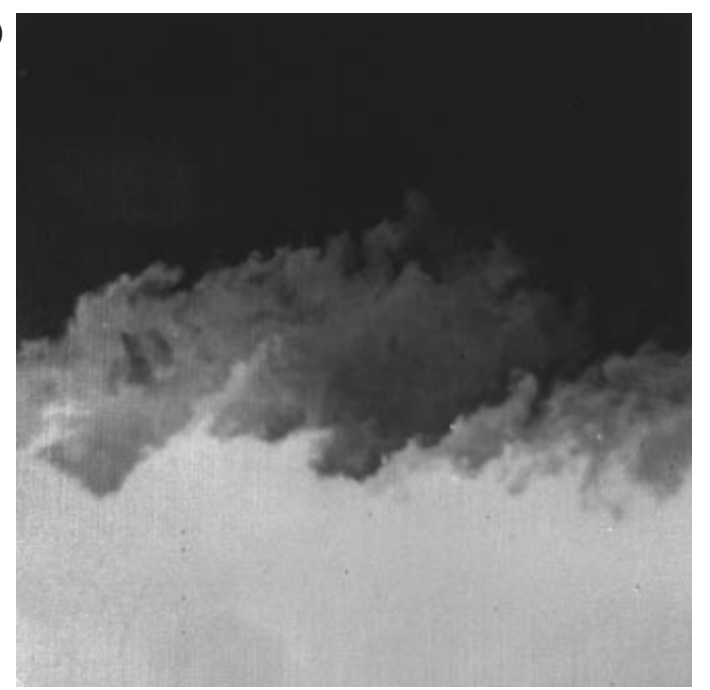

FIGURE 5. Medium-compressibility $\left(M_{1} \simeq 1.5\left[\mathrm{~N}_{2}\right], M_{2} \simeq 0.3\left[\mathrm{C}_{2} \mathrm{H}_{4}\right], M_{\mathrm{c}} \simeq 0.54\right)$ shear-layer flow. (a) Schlieren image (see text). (b) Rayleigh-scattering scalar-field image, corrected for aero-optic effects.

the homogeneity in the mixed fluid composition is much reduced at higher compressibility, corroborating the reduction in the dynamical significance of large-scale vortical-motion transport and mixing at higher compressibility. These inferences are supported by all the Rayleigh-scattering images recorded at each flow condition.

Figure 6 depicts a schlieren $(a)$ and a (corrected) Rayleigh-scattering $(b)$ image recorded at the highest compressibility conditions in these experiments $\left(M_{1} \simeq 1.5[\mathrm{He}]\right.$, $M_{2} \simeq 0.3\left[\mathrm{C}_{2} \mathrm{H}_{4}\right], U_{2} / U_{1} \simeq 0.09, \rho_{2} / \rho_{1} \simeq 4.1, M_{\mathrm{c}} \simeq 0.96$ ). Lab-frame Mach waves in the $M_{1} \simeq 1.5$ high-speed free stream, as well as stronger (propagating) oblique waves in the low-speed free stream can be seen (approximate extent of Rayleigh-scatteringimage field of view also indicated). Also registered is the (smaller) convergence angle for the bottom guidewall, corresponding to the lower entrainment requirements for this shear layer. For this flow, the sensitivity of the visualization to high-speed free-stream fluid concentrations is substantially lower, owing to the (much) smaller Dale-Gladstone index-of-refraction constant for helium. The schlieren optical system sensitivity was accordingly increased to record the (weak) waves in the high-speed free stream. To maintain a similar visualization contrast between the top and bottom parts of the image, the bottom (low-speed) free-stream composition was changed to a mixture of nitrogen and ethylene (recall that these are density matched), to adjust the scaling between number-density gradients and index-of-refraction gradients in the two free streams. This allowed the Mach waves (including the ones emanating from the downstream end of the aerodynamic window) to be visible, along with the 
(a)

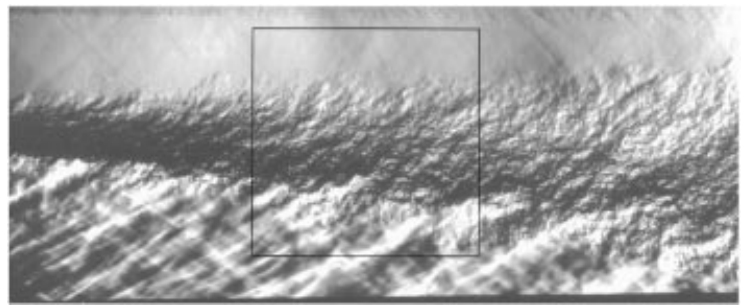

(b)

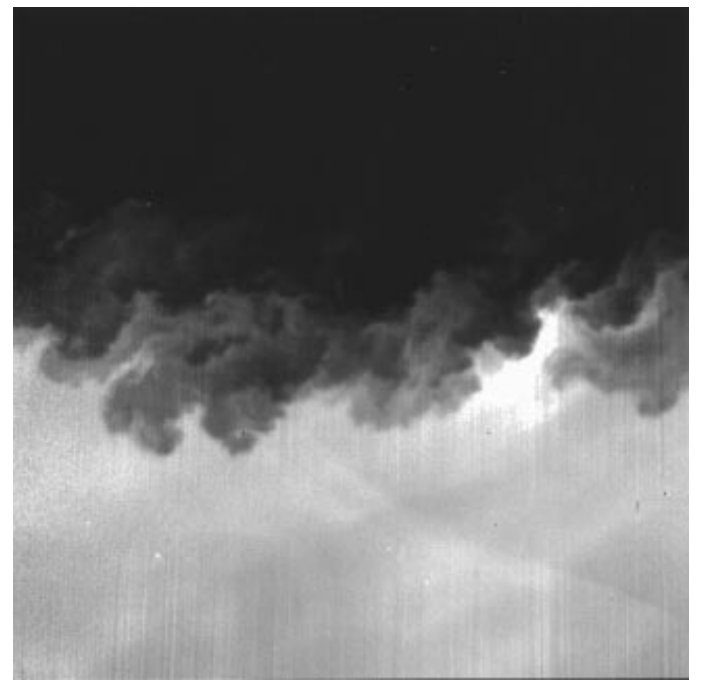

FIGURE 6. High-compressibility $\left(M_{1} \simeq 1.5[\mathrm{He}], M_{2} \simeq 0.3\left[\mathrm{C}_{2} \mathrm{H}_{4}\right], M_{\mathrm{c}} \simeq 0.96\right)$ shear-layer flow. $(a)$ Schlieren image (see text). (b) Rayleigh-scattering scalar-field image, corrected for aero-optic effects.

(stronger) waves in the low-speed free stream. Again, flow disturbances in the highspeed-stream (top) boundary-layer region generated by the aerodynamic window are not discernible.

The oblique wave system in the bottom free stream is generated by flow disturbances in the turbulent shear-layer region convecting with a speed that is higher than that of sound in the (subsonic) low-speed free stream. They have been observed previously in similar supersonic/subsonic free-stream experiments in this facility and used to estimate the convective Mach number with respect to the low-speed free stream (16) from the angle of the oblique waves (Hall et al. 1993),

$$
M_{\mathrm{c} 2} \equiv \frac{U_{\mathrm{c}}-U_{2}}{a_{2}}=2.2-2.5 .
$$

Two families of oblique waves can be seen, corresponding to waves generated directly and by (near-) specular reflection from the bottom guidewall. They can be thought of as a succession of 'sonic booms' generated by pressure disturbances in the shear-layer region, moving supersonically with respect to the (subsonic) low-speed free stream. This corresponds to a convection velocity, $U_{\mathrm{c}}$, for the turbulent shear-layer structures that is asymmetric and, in particular, relatively close to the top-free-stream speed, $U_{2}$. This asymmetry was noted experimentally by Papamoschou (1991) and attributed to asymmetries in total pressure at convecting stagnation points that can be sustained because shocklets can form with respect to one side only (Papamoschou 1991). A 
quantitative model of this process yielding results for $U_{\mathrm{c}}$ in good agreement with the Papamoschou and other available data, as well as the estimate in (18) above, was put forth by Dimotakis (1991a). In this model, the required total-pressure losses need not be dissipated across a single shock. Direct experimental evidence of single, internal shocks of the requisite strength to generate such asymmetries has not been documented and they were not captured in the present investigation.

It is interesting to compare the data in the schlieren image to those in the (corrected) Rayleigh-scattering image slice (figure 6b). For a helium/ethylene shear layer, the imaged Rayleigh-scattered intensity is (very nearly) proportional to the number density of ethylene (indicated by grey-scale value). The Rayleigh-scattering image has captured the oblique wave pattern in the imaged plane. The strength of these waves can be assessed in terms of the density increase behind the compression waves, or more accurately, in terms of the wave-angle change of the reflected waves, as they interact with the primary waves. As can be seen, the number density of ethylene can exceed the (undisturbed) free-stream number density. A conspicuous (bright) free-stream feature, adjoining the edge of the mixed-fluid region, corresponds to a density increase behind the oblique compression wave of $\Delta \rho / \rho=0.20-0.25$. For room-temperature ethylene $(\gamma \simeq 1.24)$, this corresponds to a (normal-shock) Mach number of $M_{\mathrm{s}}=1.14-1.18$. This is the estimated local shock strength, as would be registered in the free-stream gas. Higher shock strengths are likely in the shear-layer interior, in particular, prior to interactions with accompanying expansion fans, which will weaken and turn the shocks (e.g. Dimotakis 1991a, figure 10b).

The Rayleigh-scattering image at high compressibility supports the same progression in behaviour between low- and medium-compressibility flows. Organized, largescale vortical motion across the full transverse extent of the mixing-layer region is less clear yet. Nevertheless, mixed fluid can be seen to be bounded by relatively sharp irregular interfaces. Unfortunately, the negligible Rayleigh-scattering cross-section of helium $(10 b)$ renders visualization of mixed fluid whose composition is dominated by helium effectively infeasible by this means.

The progression in compressibility (increasing $M_{\mathrm{c}}$ ) in these experiments was accompanied by variations in the shear-layer free-stream density ratio, $\rho_{2} / \rho_{1}$. This is an important parameter in shear-layer dynamics and, as a consequence, not all the changes observed may be attributable to compressibility. Conversely, the progression in free-stream density ratio is not monotonic $\left(M_{\mathrm{c}} \simeq 0.15,0.54,0.96\right.$; with $\rho_{2} / \rho_{1} \simeq 1.0$, $0.68,4.1$, respectively), whereas the decreasing flow coherence is monotonic with increasing $M_{\mathrm{c}}$. Finally, we note that shear-layer flows with a supersonic high-speed free stream (nitrogen and helium, respectively) were characterized by roughly twice the value of the local Reynolds numbers, i.e. $R e_{\delta} \approx 10^{6}$.

Flows at medium compressibility, $M_{\mathrm{c}} \simeq 0.54$ (and high Reynolds number) produced less aero-optical distortions than the other two flow conditions studied. The imagecorrection scheme was also the most successful for these flows. This can be seen (figure 5) by comparing the uniformity in the low-speed free-stream fluid region (after corrections). Sampling a small image portion in that free stream, the r.m.s. deviation of the measured intensity is found to be $\lesssim 1 \%$. This can be compared to the estimated number of $1.5-2.0 \times 10^{4}$ (peak) detected photons/pixel (1500-2000 $\mathrm{A} / \mathrm{D}$ units $(\mathrm{ADU})$, at $\approx 10 \mathrm{e} / \mathrm{ADU}$, for this CCD camera), corresponding to image detection very close to the photon shot-noise limit in ethylene.

Under other flow conditions, variations in detected free-stream values were dominated by residual aero-optics streaks, as can be ascertained in the corrected images in figures 4 and 6 . Those, in turn, result from rays refracted away from the original 


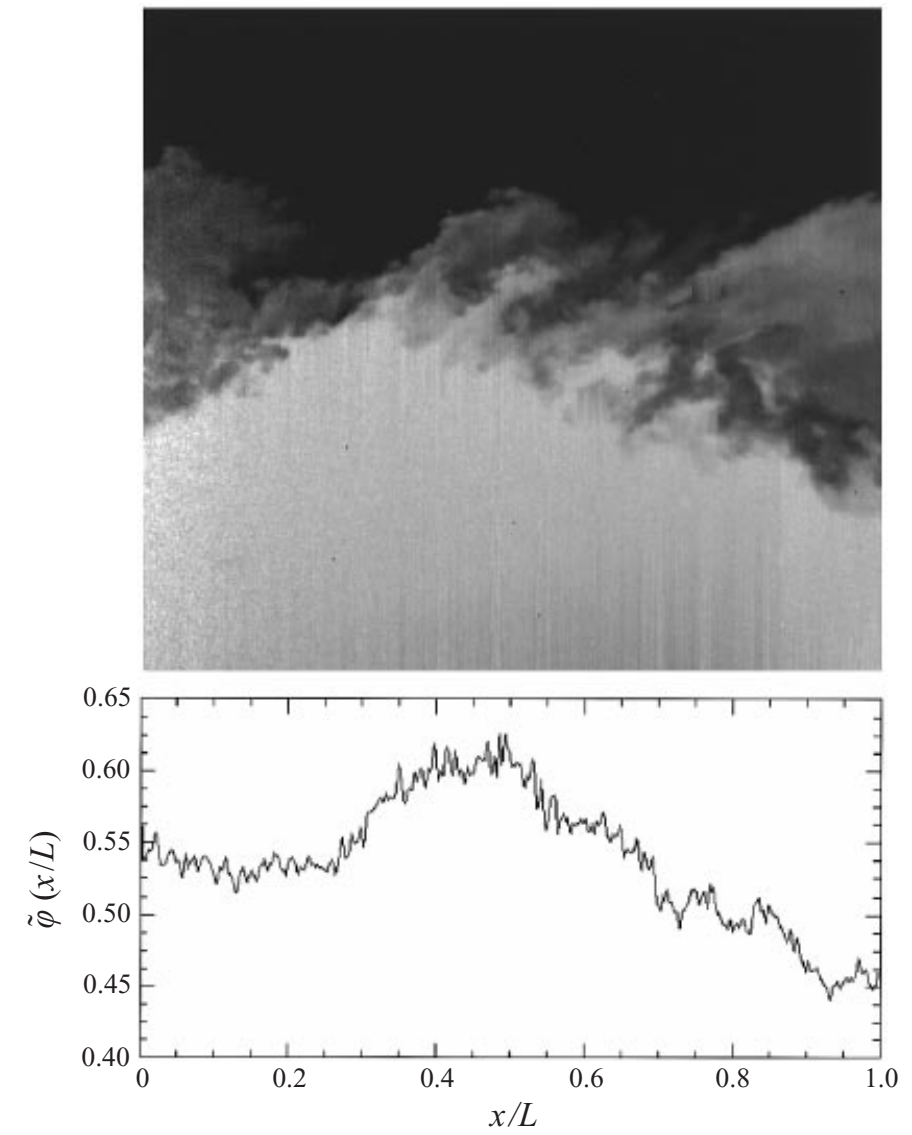

FIGURE 7. Low-compressibility (subsonic, $M_{\mathrm{c}} \simeq 0.15$ ) shear-layer index-of-refraction image and normalized wavefront-phase integral, $\tilde{\varphi}(x / L)$, as given by (4), computed for a subsonic, shear-layer realization (data in figure 4).

laser-sheet fan direction(s). Recall that the correction scheme employed (17) assumes no refractive changes in ray (wavefront-normal) propagation direction, to leading order. Inspection of the incompressible-flow image in figure 4 reveals a relatively uniform, fuzzy horizontal band in the low-speed free stream, roughly, 0.15-0.2 of the height from the bottom, corresponding to the location of the reference strip used for the aero-optical correction. The scheme is quite successful there, with faint residual streaks visible above and below this strip. The slight variations in the diffracted ray (streak) directions can be discerned by sighting along them.

\subsection{Wavefront-phase behaviour}

Wavefront-phase function calculations were performed, as described in the Introduction, on the corrected two-dimensional index-of-refraction fields. A sample is presented in figure 7. It depicts the scaled wavefront-phase function, $\tilde{\varphi}(x / L)$, as given in (4), based on the data in figure $4(b)$, reproduced here $(a)$ for reference.

Scaled wavefront-phase integrals were computed across the imaged field of view, with $L$ equal to the extent of the imaged field (here, $L \approx 2 \delta$, i.e. roughly, twice the local shear-layer width) and $n_{\infty}=n_{1}$ the (low) index of refraction of the high-speed fluid 

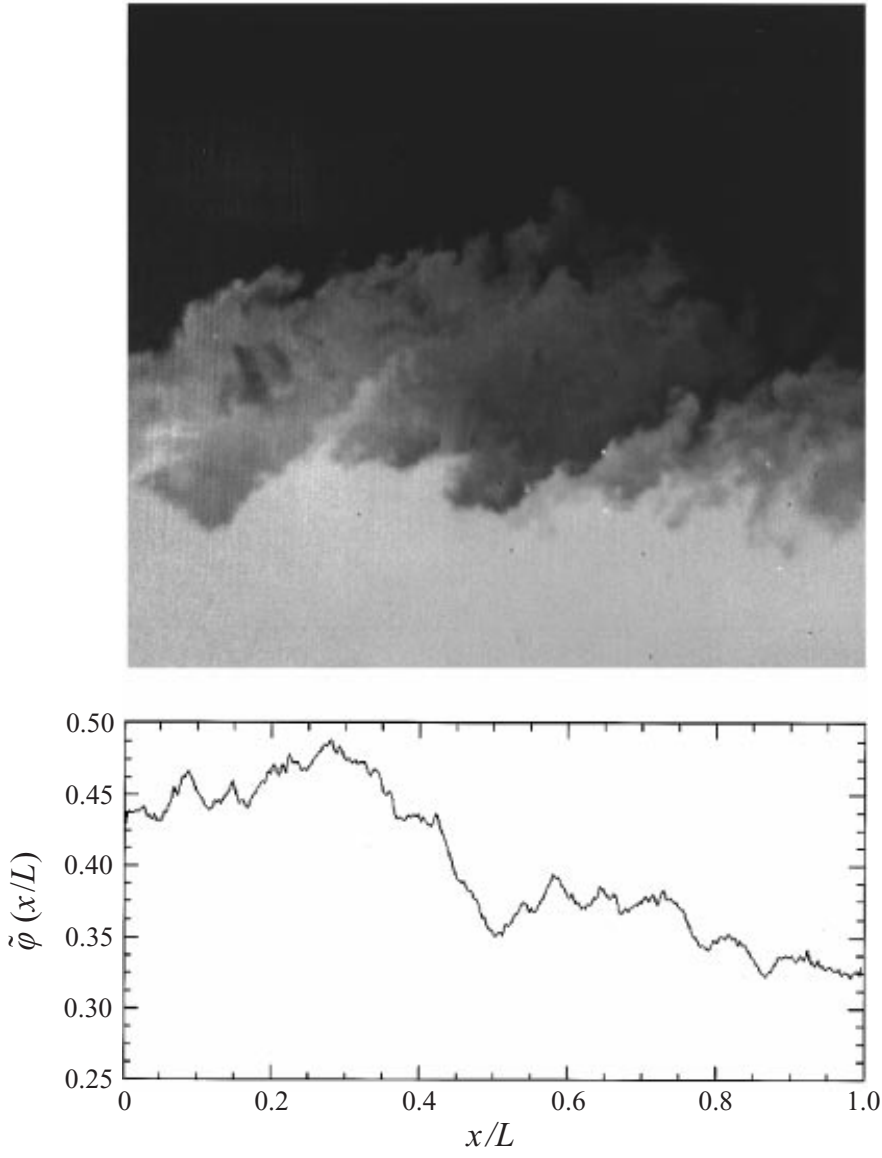

FIGURE 8. Moderate-compressibility $\left(M_{1} \simeq 1.5 \mathrm{~N}_{2}, M_{\mathrm{c}} \simeq 0.54\right)$ shear-layer data and corresponding normalized wavefront-phase integral, $\tilde{\varphi}(x / L)$. Scalar-field data from figure 5 .

(figure 7). Similar calculations, computed for the higher-compressibility realizations, are reproduced in figures 8 and 9.

The resulting wavefront-phase functions show that the shear-layer large-scalestructure organization is responsible for the largest effects, as also noted by Fitzgerald \& Jumper (1998) in their experiments on high-Re shear layers. Truman \& Lee (1990) also noted the dominant influence of large-scale structures in simulated optical propagation through computed turbulent flow. The wavefronts are seen to generate left- and right-steering optical wedges (prisms), to lowest order, corresponding to locally positive- and negative-streamwise-gradient regions, as well as locally positive and negative lenses, corresponding to local maxima and minima in the wavefrontphase function. Positive lenses can produce focusing, or caustics, behind the turbulent optical-distortion region. Such caustics were directly imaged previously in elevatedpressure experiments on turbulent jets (Fourguette et al. 1995; cf. also Tyson 1991, p. 26). Effective negative lenses will diverge the beam. All three effects will remove beam energy from a receiving aperture some distance away or, in the case of positive lensing resulting in caustics in the intervening distance between the distorting turbulent region and a receiver, render it effectively infeasible to compensate for phase errors using adaptive optics. 

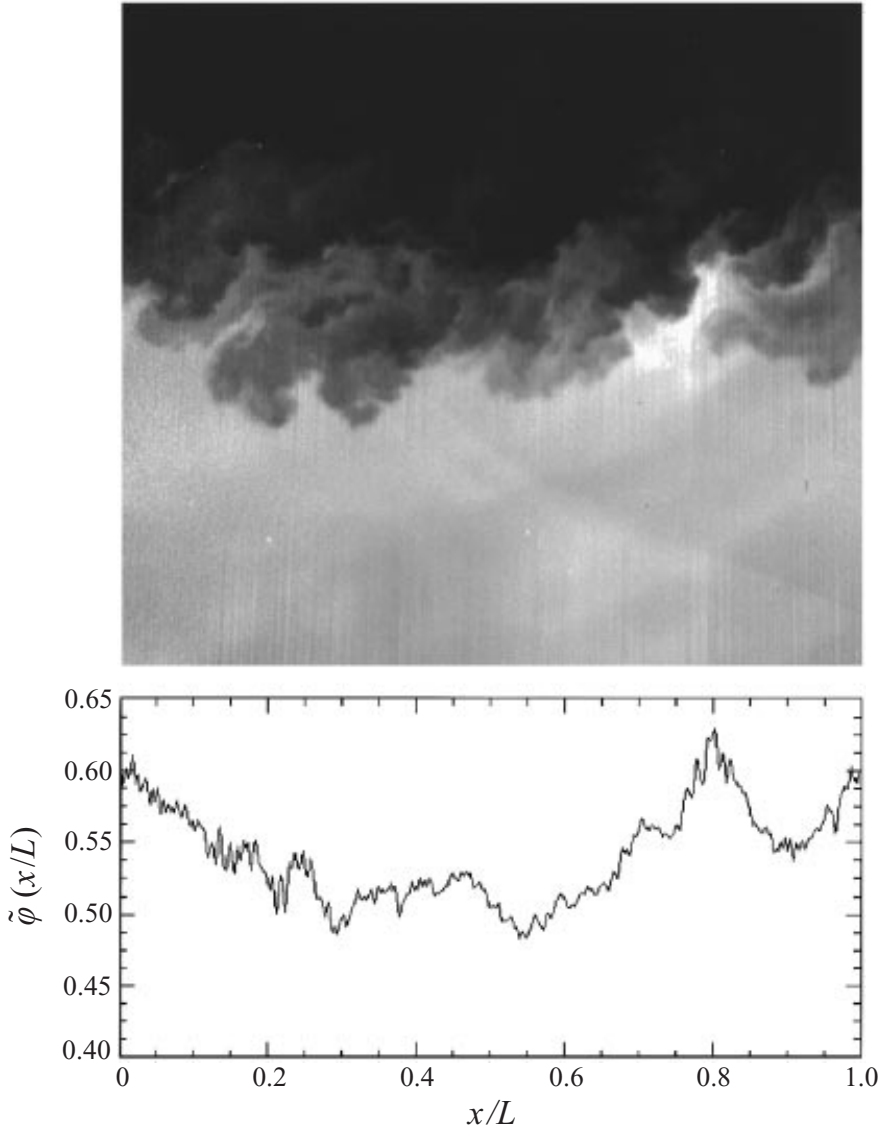

FIGURE 9. High-compressibility $\left(M_{1} \simeq 1.5 \mathrm{He}, M_{\mathrm{c}} \simeq 0.96\right)$ shear-layer data and corresponding normalized wavefront-phase integral, $\tilde{\varphi}(x / L)$. Scalar-field data from figure 6 .

For this propagation direction, global shear-layer aero-optical effects will depend on the transverse extent of the optical beam. If the transverse extent subtends several large-scale structures, the shear layer will look like a continuously variable phase grating, with a characteristic local spatial scale given by the large-scale vortical structures. For a beam subtending several structures, the resulting convected phase grating will have a spatial pitch that is linearly evolving (in the mean) in the streamwise direction. For a temporally growing shear layer, as might be more appropriate in an atmospheric jet stream, for example, the flow will act like a convected phase grating, with a more-or-less-uniform spatial pitch in the streamwise direction. For a beam whose transverse extent subtends, roughly, a single large-scale structure, the aerooptical effect should be describable as that of a set of (convecting) optical wedges and lenses. A beam of much smaller spatial extent than a large-scale structure will be dominated by smaller-scale index-of-refraction-field fluctuations. This behaviour will be further discussed below, in the context of a simple model of shear-layer/opticalbeam interactions.

Ensemble-averaged spatial spectra of the scaled wavefront-phase functions, $\tilde{\varphi}(x / L)$, were also computed as Fourier transforms of the $\tilde{\varphi}(x)$ spatial correlation functions, for the three flow conditions described above, after windowing the data with 1/4-period 


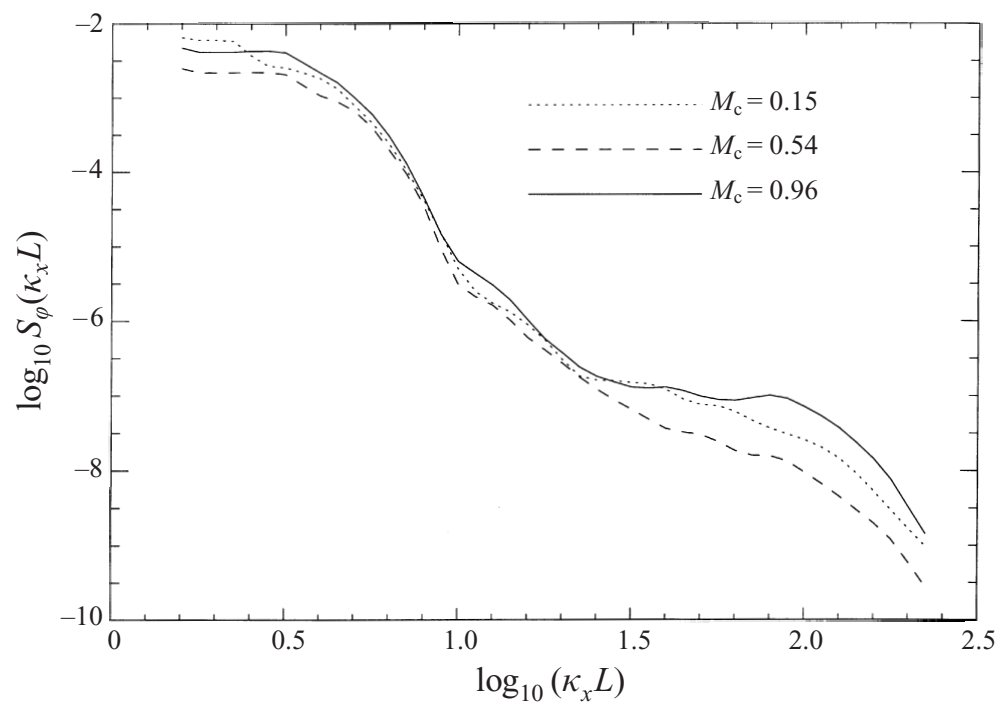

FIGURE 10. Ensemble-averaged spectra of scaled wavefront-phase functions, $\tilde{\varphi}(x / L)$, for shear layers of variable compressibility: $M_{\mathrm{c}}=0.15,0.54$, and 0.96 .

sines/cosines for the first and last 1/8-record intervals. These are plotted in figure 10 . Lines of increasing solidity denote increasing compressibility.

The spectra are characterized by a near-power-law region at intermediate wavenumbers, where $S_{\varphi}\left(\kappa_{x} L\right) \propto\left(\kappa_{x} L\right)^{-q}$, with $q \simeq 2$. They also provide an independent indicator of the dynamic range of the image data, spanning $\approx 7$ decades in signal power $(\approx 3.5$ decades in amplitude $) \cdot \dagger$

The spatial wavefront-phase-function spectra can be seen to be (weakly) sensitive to compressibility, mostly at the high-wavenumber end of the spectrum, with a nonmonotonic influence of increasing $M_{\mathrm{c}}$, at least in the range of flows investigated. In particular, the lowest spectral values at high wavenumbers are encountered for the moderate (intermediate) compressibility case. This may well be attributable to a combination of Mach-number, Reynolds-number, and density-ratio effects, which need not act in the same way.

Recall also that, under these flow conditions, ray diffractions were at a minimum, permitting a relatively more successful application of the aero-optical correction scheme. The lower spectral contributions at high wavenumbers are evident, directly, in the scalar-field images and computed phase-front functions. Similar behaviour, as regards Mach-number and Reynolds-number combinations, has been observed in direct measurements of molecular mixing in chemically reacting shear layers (Slessor 1998).

Streamwise phase-front-tilt functions,

$$
\psi(x / L)=L \frac{\partial \tilde{\varphi}}{\partial x}=\frac{L}{\Delta n} \int \frac{\partial n}{\partial x} \mathrm{~d}\left(\frac{z}{L}\right)=\frac{1}{\Delta n} \int \frac{\partial n}{\partial x} \mathrm{~d} z,
$$

were also computed. As expected, their spectra are nearly wavenumber-independent

$\dagger$ It might appear that this dynamic-range is inconsistent with the estimated $\approx 1 \%$ r.m.s. amplitude in the pure $\mathrm{C}_{2} \mathrm{H}_{4}$ free stream. The phase-front functions, however, are computed as integrals (sums) over image columns spanning 500 pixels. 
(nearly white), since

$$
S_{\psi}\left(\kappa_{x} L\right)=\mathscr{F} \mathscr{T}\left\{<\psi\left(x^{\prime}\right) \psi\left[\left(x-x^{\prime}\right) / L\right]>_{x^{\prime}}\right\} \propto\left(\kappa_{x} L\right)^{2} S_{\varphi}\left(\kappa_{x} L\right) .
$$

\section{Beam propagation through turbulent jets}

Aero-optical effects in the near and intermediate fields $\left(x / d_{\text {jet }} \lesssim 15\right)$ of gas-phase turbulent jets were also investigated over a modest range of Reynolds numbers. The jet experiments were conducted in the GALCIT High-Pressure Combustion Facility (HPCF; Gilbrech 1991). They are a sequel to previous related investigations in the same facility (Fourguette et al. 1995), over a range of pressures, with various modifications and improvements. They entailed uniform-density, axisymmetric jets with the same $\mathrm{C}_{2} \mathrm{H}_{4} / \mathrm{N}_{2}$ gas pair. In the present experiments, however, $\mathrm{N}_{2}$ was used as the jet fluid, discharging into $\mathrm{C}_{2} \mathrm{H}_{4}$, to facilitate the direct imaging of the aero-optical effects beyond the jet, using the high- $\sigma$ ambient scattering environment afforded by the $\mathrm{C}_{2} \mathrm{H}_{4}$.

\subsection{Experiments and scalar-field structure}

In the context of Rayleigh-scattering measurements, there is an advantage in raising pressure. In a constant-temperature environment, the number density of scatterers and, therefore, the Rayleigh-scattered signal intensity, increases linearly with pressure, with signal-to-noise ratios (SNRs) for a photon shot-noise-limited measurement increasing as the square root of pressure. Conversely, index-of-refraction gradients also increase linearly with pressure and the ability to image through the turbulent, wavefront-phasedistorting medium is degraded. The degradation due to the latter was found to be acceptable up to ambient pressures of 8-10 atm (Fourguette et al. 1995). Improvements in image detection in the interim (e.g. use of a lower-noise, higher-quantum-efficiency, back-illuminated $\mathrm{CCD}$ ) permitted a lowering of the ambient pressure while matching, or exceeding, the previously achieved image SNRs. The pressure for all experiments reported here is $4 \mathrm{~atm}$.

Examples, recorded at jet Reynolds numbers, $R e \simeq 9.0 \times 10^{3}, 18 \times 10^{3}$, and $30 \times 10^{3}$, where here $R e=U_{\text {jet }} d_{\text {jet }} / v_{\text {jet }}$, with $d_{\text {jet }}$ the jet diameter, $U_{\text {jet }}$ the jet nozzle velocity, and $v_{\text {jet }}$ the jet-fluid viscosity, are shown in figures 11-13. The edges of the laser sheet, which facilitated the location of the virtual origin, are discernible. The selected range of Reynolds numbers spans the mixing transition (Dimotakis 2000), at $R e_{\mathrm{tr}} \simeq 1-2 \times 10^{4}$, and is in the range investigated earlier in both gas-phase and liquid-phase turbulent jets - liquid-phase $S c \approx 2.0 \times 10^{3}, v s$. gas-phase $S c \simeq 1$ here (Dowling \& Dimotakis 1990; Miller \& Dimotakis 1991; Catrakis \& Dimotakis 1996). The previous studies cited were in the jet far field, i.e. $x / d_{\text {jet }}>30$, however. In the jet-data figures presented here, the (inverted) grey-scale in the jet region (white-to-black) denotes decreasing jetfluid (nitrogen) mole fraction (11). This placed the high-index-of-refraction medium on both sides of the jet, permitting more precise aero-optical corrections to be carried out, as will be described below. The streaks below the jet are the aero-optical counterparts of the ones encountered in the shear-layer flows discussed above.

The high-index-of-refraction fluid on both sides of the turbulent region precludes use of the same image-correction scheme as used in the shear-layer experiments; a different image-processing method was devised. Following pixel-by-pixel background subtraction (\$2), with ensembles of eight background (empty-tank) images, laser-sheet non-uniformities are removed by normalizing with an ensemble of eight images of pure $\mathrm{C}_{2} \mathrm{H}_{4}$ gas at the same pressure (flat-fielding). The data in figures 11-13 are 


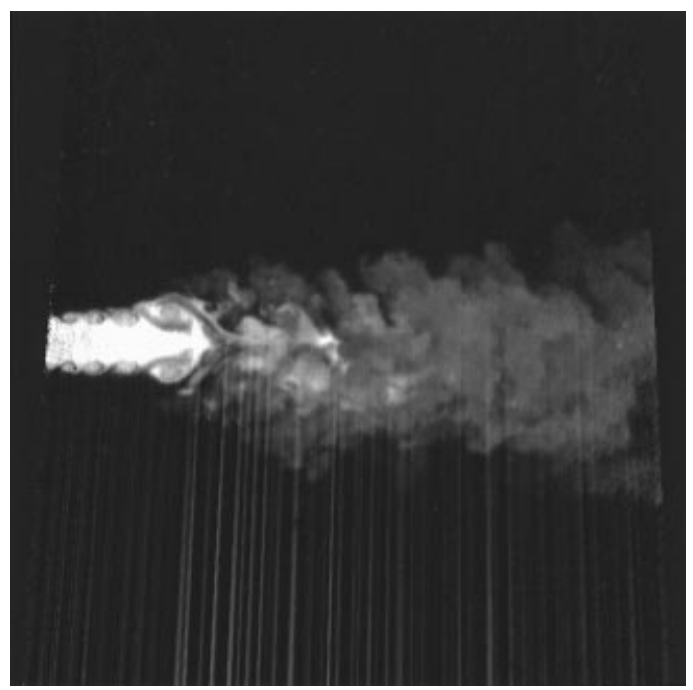

FIGURE 11. Image of $\mathrm{N}_{2}$ jet into $\mathrm{C}_{2} \mathrm{H}_{4}$, at $\mathrm{Re} \simeq 9.0 \times 10^{3}$ and $p \simeq 4 \mathrm{~atm}$ (inverted grey-scale denotes imaged scattering intensity). Beam propagation is top-to-bottom in the image. Aero-optical streaks, caused by index-of-refraction variations, are evident below the jet.

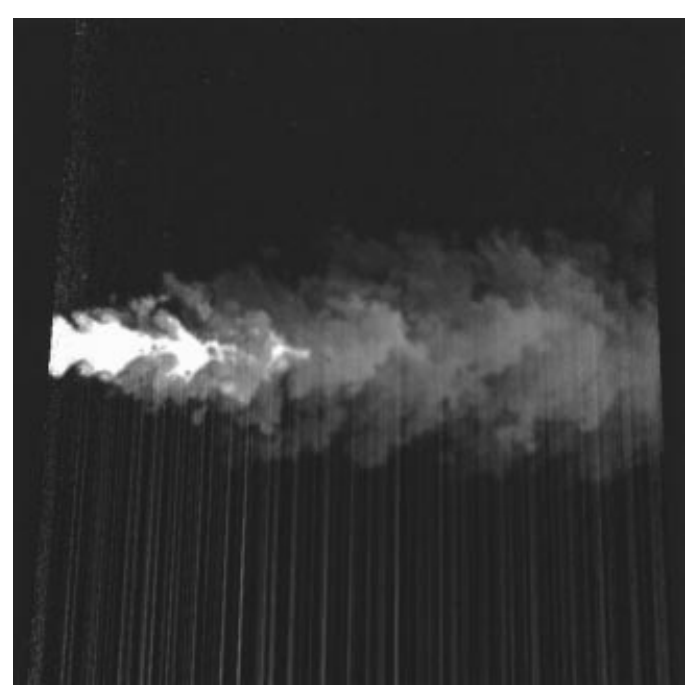

FIGURE 12. As figure 11 but at $R e \simeq 18 \times 10^{3}$.

corrected by this process and represent an intensity field given by (angle brackets denote ensemble averages, cf. Catrakis \& Dimotakis 1996)

$$
I(x, z)=\frac{I_{\text {raw }}(x, z)-\left\langle I_{\text {back }}(x, z)\right\rangle}{\left\langle I_{\text {ill }}(x, z)\right\rangle-\left\langle I_{\text {back }}(x, z)\right\rangle}
$$

The jet data in figures 11-13 were further corrected for the aero-optical streaks using spatial (complex) Fourier filtering, after first being transformed into polar coordinates about the virtual origin of the laser sheet. A typical two-dimensional (polarcoordinate) spatial power spectrum, computed after smooth (1/8-record, quarter- 


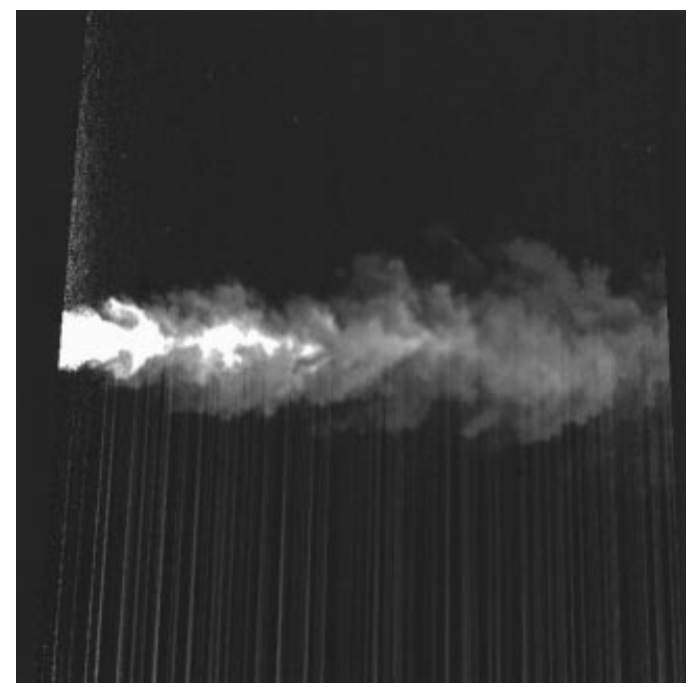

FIGURE 13. As figure 11 but at $R e \simeq 30 \times 10^{3}$.

(a)

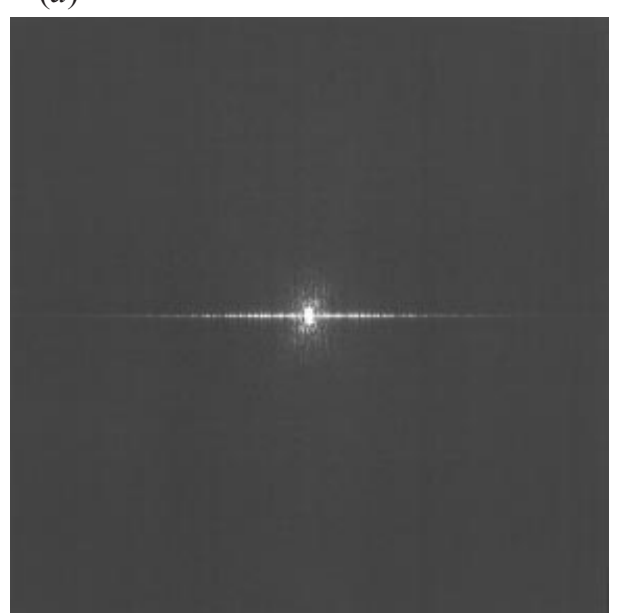

(b)

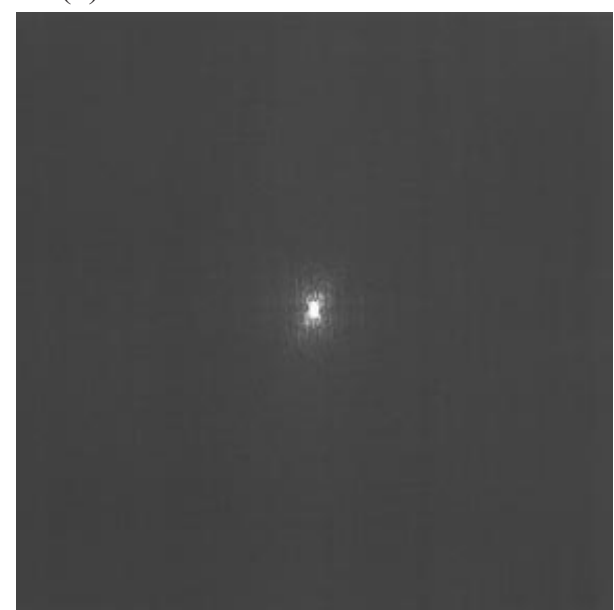

FiguRE 14. Example of a two-dimensional spatial scalar power spectrum at $R e \simeq 9.0 \times 10^{3}$. (a) Total spectrum. (b) Notch-filtered spectrum.

sine/cosine) windowing at the image boundaries, is shown in figure 14(a), computed for the $R e \simeq 9 \times 10^{3}$ jet data realization (figure 11).

The narrow spectral-energy band in figure $14(a)$ can be seen to be closely aligned with the $\kappa_{r}$-axis, marking the contribution of the radial aero-optical streaks in the $(r, \theta)$-plane. This is the reason for the coordinate transformation, which removes directional variations (ray fan) associated with the spreading of the incoming laser sheet (cf. figure 11 and discussion in $\S 3$ on beam propagation in shear layers). A notch filter is then applied that removes the thin spectral band straddling the $\kappa_{r}$ axis, excluding a small region near the origin. The removed Fourier components are replaced with locally interpolated components, computed using data at the boundaries of the notch region. All filtering operations are performed in complex-Fourier space 

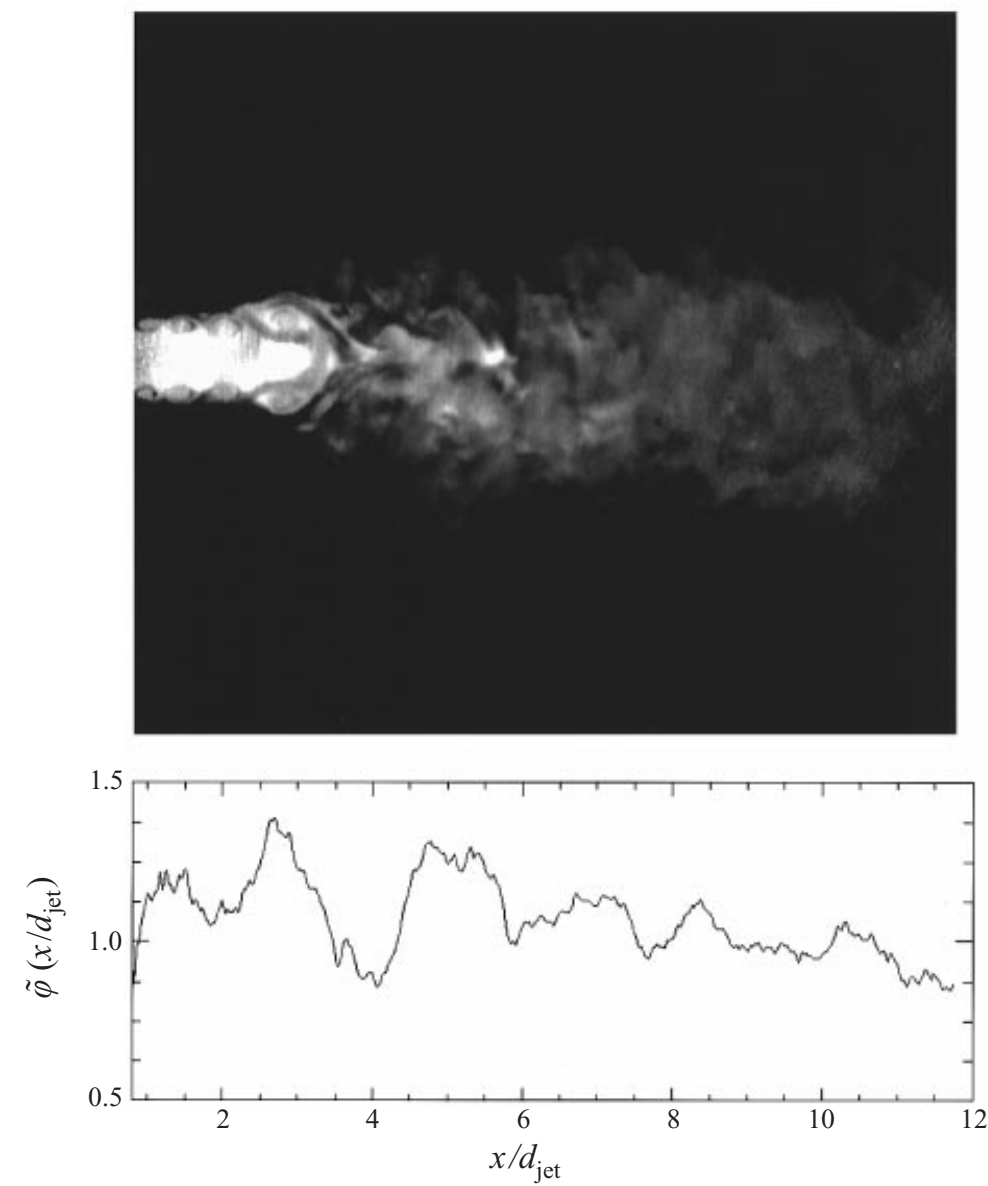

FIGURE 15. Fourier-filtered image at $R e \simeq 9.0 \times 10^{3}$ (cf. figure 11) and corresponding scaled wavefront-phase function profile.

permitting the phase of the Fourier filter to properly discriminate between the two (top/bottom) half-spaces.

The resulting, notch-filtered spectrum for these data is shown in figure 14(b). A complex inverse-Fourier transform, followed by a transformation back from polar to Cartesian coordinates, is then performed to yield the corrected, 'streak-free' images. Processed jet-realization images using this technique are shown in figures $15-17$, for the data shown above (cf. figures 11-13).

\subsection{Wavefront-phase behaviour}

The Fourier-filtered jet-image data were used to compute the scaled wavefrontphase-function integrals along the $z$ (top-to-bottom) beam-propagation direction. In the expression for the wavefront-phase function (4), as applied to the jet data, the normalizing index-of-refraction amplitude and length scale were chosen as

$$
\Delta n=\left|n_{\text {jet }}-n_{\infty}\right|
$$

using the jet diameter as the characteristic length scale,

$$
L=d_{\text {jet }} .
$$



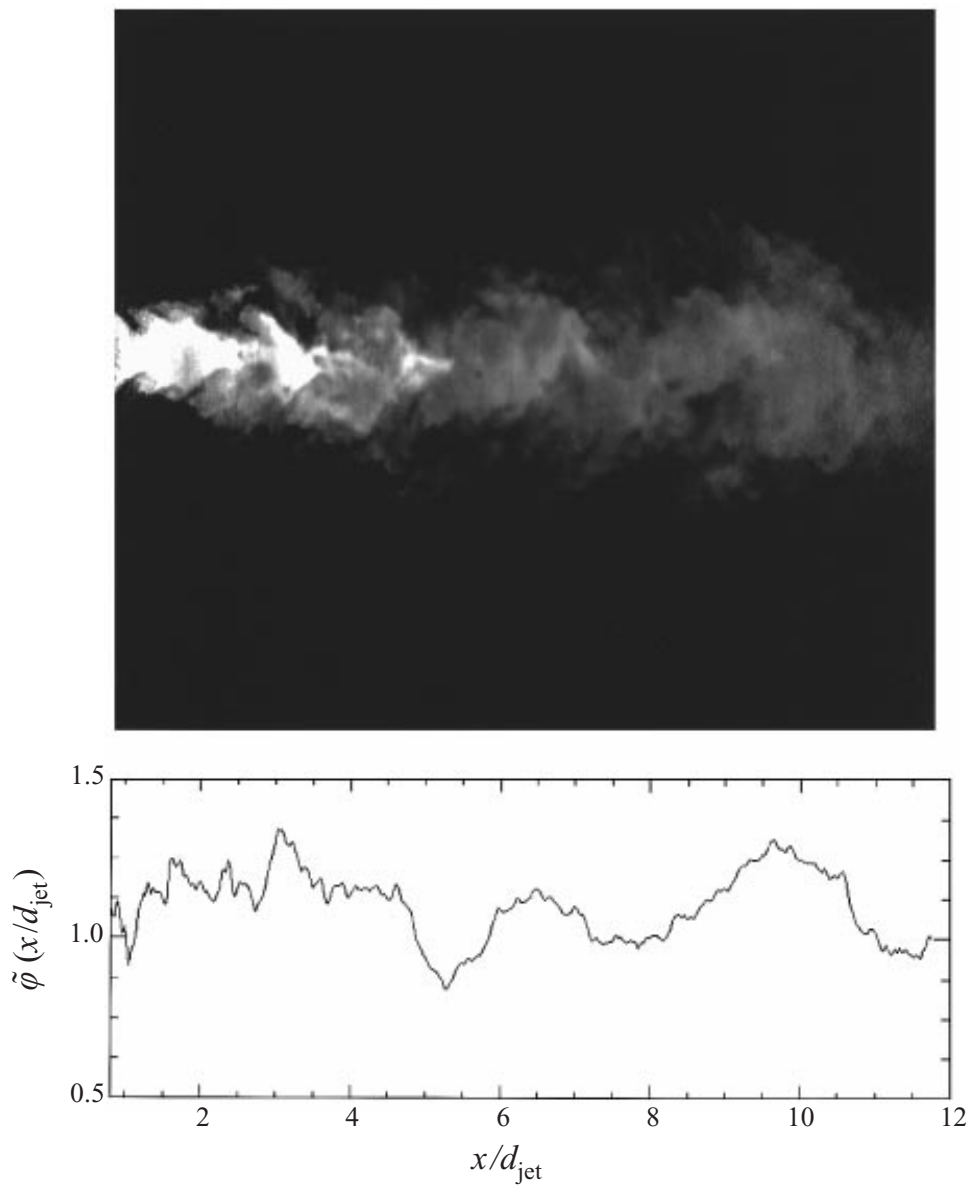

FIGURE 16. Fourier-filtered image at $R e \simeq 18 \times 10^{3}$ (cf. figure 12) and corresponding scaled wavefront-phase function profile.

Examples of the scaled wavefront-phase functions, $\tilde{\varphi}\left(x / d_{\text {jet }}\right)$, computed from realizations at each of the three Reynolds numbers, i.e. $R e \simeq 9.0 \times 10^{3}, 18 \times 10^{3}$, and $30 \times 10^{3}$, are also shown in figures $15-17$. They are plotted below the corresponding jet-image data, to facilitate visual comparison, as with the shear-layer data. The $x$-axis is along the jet streamwise direction and scaled in jet-diameter units. With this scaling and the scaled index-of-refraction field values, the mean scaled wavefront-phase function values are near unity and independent of the downstream coordinate, $x / d_{\mathrm{jet}}$, as expected for round, turbulent jets.

As with the shear layers, the large-scale excursions in the wavefront-phase integrals for the jets can be seen to stem from the large-scale dynamics and organization in the flow, confirming previous lower-Re studies, in the region closer to the jet nozzle (McMackin et al. 1995). The rear-to-front entrainment process of the large vortical structures produces systematic compositional variations within each largescale structure, with attendant wavefront-phase variations that do not diminish in amplitude with distance downstream, or increasing Reynolds number.

For the downstream range investigated here $\left(x / d_{\text {jet }}<15\right)$, the jet diameter remains the characteristic length and can be used as the appropriate spatial length scale 

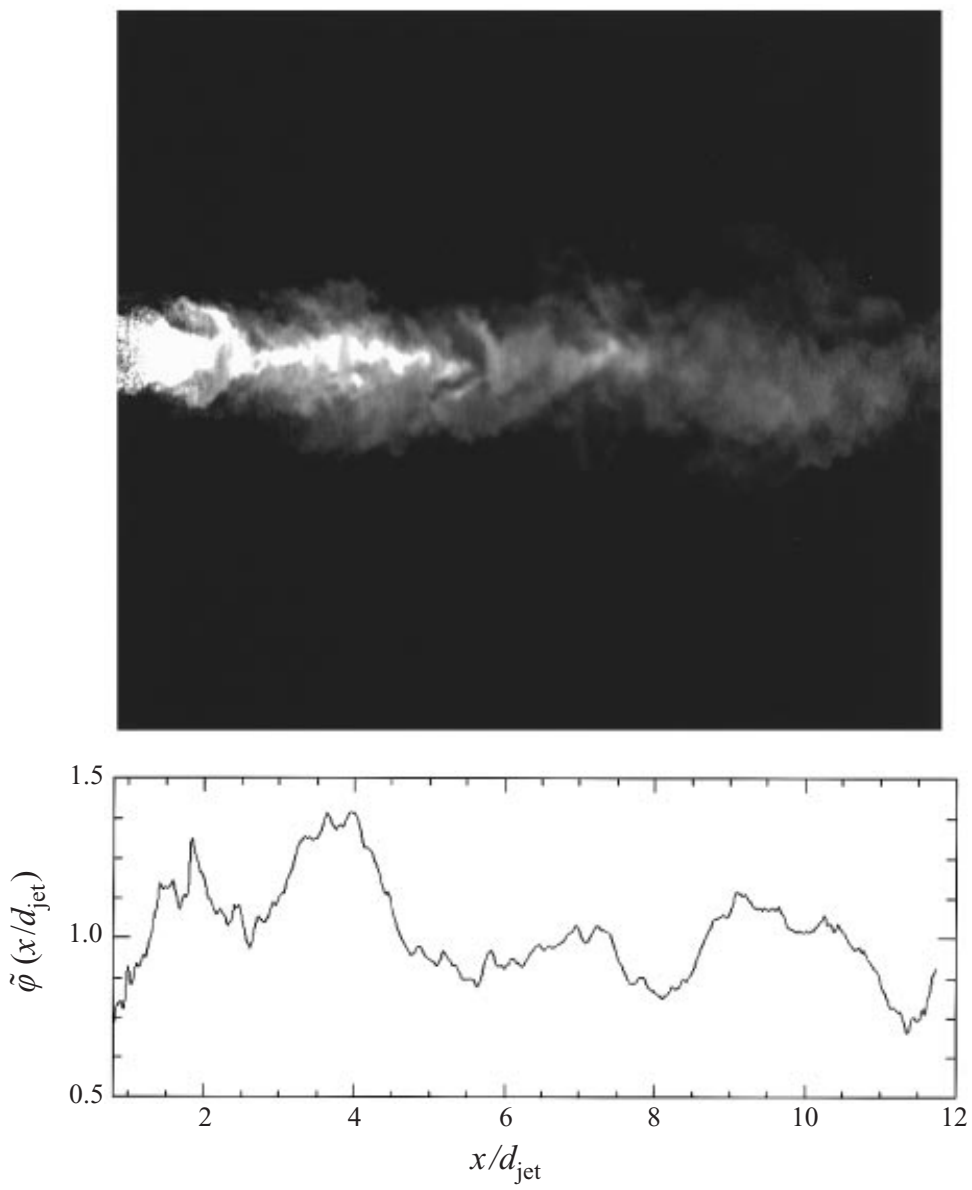

FIGURE 17. Fourier-filtered image at $R e \simeq 30 \times 10^{3}$ (cf. figure 13) and corresponding scaled wavefront-phase function profile.

throughout the spatial extent of the imaged (near-field) scalar field. For measurements that would extend to the jet far field, the appropriate scaling length would be the local turbulent-jet diameter, $\delta(x) \propto x$, which has been shown to scale the full spectrum of scalar fluctuations in the far field, in both gas-phase (Dowling \& Dimotakis 1990) and liquid-phase (Miller \& Dimotakis 1996) jets.

As with the shear-layer flows, the variable index-of-refraction field in the turbulent jet region acts as a variable-phase grating on the laser beam (sheet), with a characteristic length dictated by the local large-scale-structure size. Similar considerations would apply to optical beams whose transverse extent is larger, comparable to, or smaller than the typically large-scale-structure size, as discussed in the context of shear-layer aero-optical interactions. An important difference, of course, is that a round turbulent jet presents an optical beam with an axisymmetric field (about the jet axis), in the mean, with an expected interaction accordingly modified for a two-dimensional incident optical beam, as opposed to the one-dimensional (sheet) optical-beam geometry employed in these experiments.

The jet diameter, $d_{\mathrm{jet}}$, serves as a single scaling length in this streamwise range and permits computing scaled spatial spectra of wavefront-phase functions directly from 


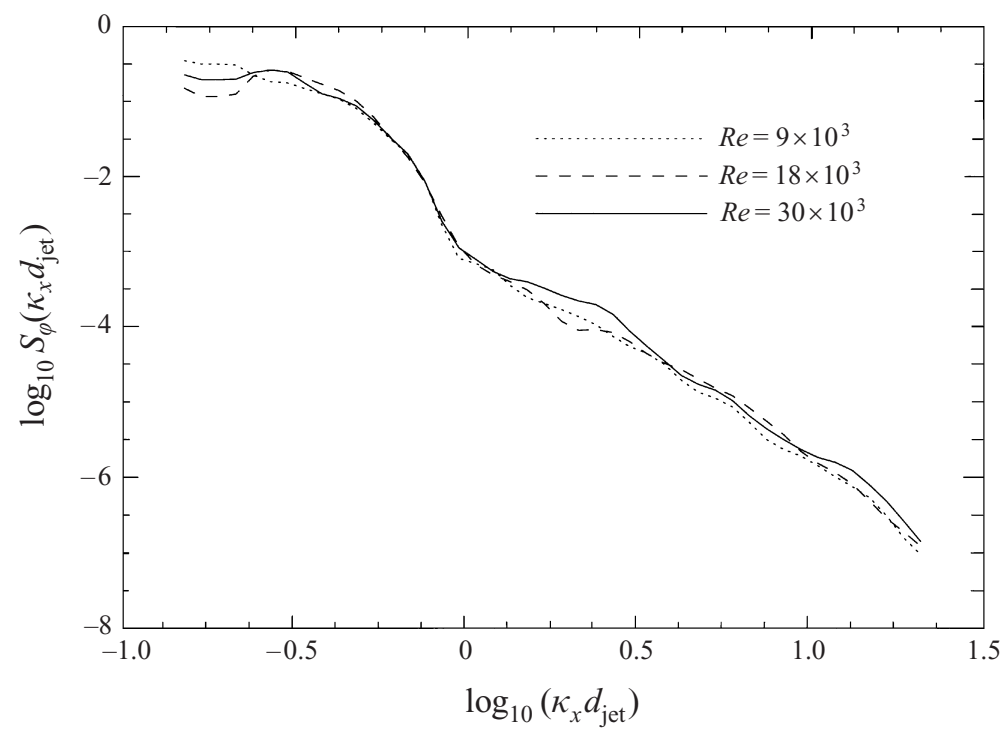

FIGURE 18. Ensemble-averaged spatial spectra of scaled wavefront-phase functions for jets at $R e \simeq 9.0 \times 10^{3}, 18 \times 10^{3}$, and $30 \times 10^{3}$.

the wavefront-phase profiles. These were calculated after 1/8-record, quarter-cosine windowing at the ends of each spatial record, as with the shear-layer data. The resulting, ensemble-averaged spectra are plotted in figure 18, vs. Reynolds number. These were computed using all the jet data recorded as part of these investigations (four images at $R e \simeq 9.0 \times 10^{3}$, three at $R e \simeq 18 \times 10^{3}$, and three at $R e \simeq 30 \times 10^{3}$ ). The wavefront-phase function spatial spectra exhibit a near power-law behaviour, $\left(\kappa_{x} d_{\text {jet }}\right)^{-q}$, at high wavenumbers $\left(\kappa_{x} d_{\text {jet }} \gtrsim 1\right)$, with $q \simeq 2.5$, i.e. steeper than for shear layers, indicating improved mixing (improved homogenization of the scalar field) in jets, relative to shear layers, at higher wavenumbers.

No statistically significant dependence on Reynolds number can be gleaned from these data, at least for this downstream extent, wavenumber, and Reynolds number range. This is in accord with previous measurements of scalar (jet-fluid) variance (fluctuation) levels, in the far field of gas-phase turbulent jets in the same Reynolds number range, in which a weak, if any, effect was registered (Dowling \& Dimotakis 1990) as opposed to a relatively strong Reynolds number effect in liquid-phase jets, in this Reynolds number range (Miller \& Dimotakis 1991; Catrakis \& Dimotakis 1996). This difference in behaviour must then be attributed to a Schmidt number effect in this Reynolds number range (Miller 1991; Dimotakis 1993).

\section{A model for beam propagation through a turbulent shear layer}

The mixed-fluid behaviour in high-Re shear layers suggests a simple model to represent the flow as regards aero-optical interactions, at least in low-compressibility (subsonic) shear layers. Specifically, mixing in low-compressibility flows at these Reynolds numbers produces large, near-uniform regions (e.g. Fiedler 1975; Konrad 1976), as also evident in the (incompressible) shear-layer data in figure 4, for example, at a prevalent composition largely dictated by the local shear-layer entrainment ratio (Dimotakis 1986, 1991b). In this environment, the main aero-optical effects will be dominated by the geometrical properties of the interfaces between the mixed- 


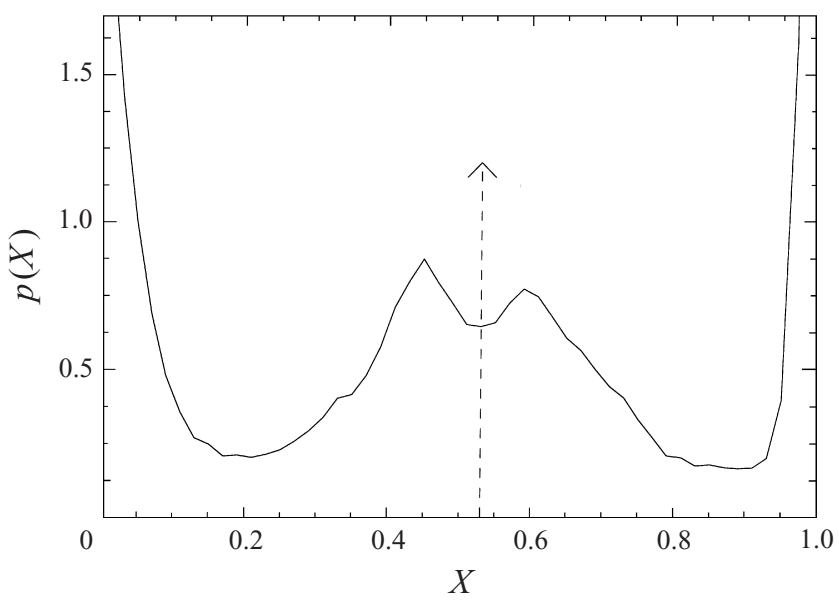

FIGURE 19. Histogram of high-speed-fluid mole fraction, $X$, for a subsonic $\left(M_{\mathrm{c}} \simeq 0.15\right)$, high-Re shear layer, computed from data in figure 4.

fluid region and the two free streams, rather than scalar (index-of-refraction) field fluctuations within the turbulent region proper.

In this context, we can explore how well the scalar field can be represented by a uniform-composition region, with level-set (isoscalar) contours separating it from each of the two free streams. This description of a high-Reynolds-number shear layer appears in Konrad (1976) and provided the basis for, or an important component in, simple models of shear-layer mixing (Broadwell \& Breidenthal 1982; Broadwell \& Mungal 1991; Dimotakis 1991b).

The scalar values for the two boundary level sets employed derive from the scalarfield histogram, which, if ensemble-averaged over many realizations, would approach the composition probability-density function (p.d.f.). Figure 19 depicts the histogram of the high-speed-fluid mole fraction, $p(X)$, estimated from the image data depicted in figure 4 . The two boundary (off-scale) peaks at either end represent the pure low- and high-speed free-stream fluids, at $X \simeq 0,1$, respectively, while the main body represents the probability of a particular mixture fraction in the mixed fluid, at least as resolved (statistically and otherwise) in these measurements.

The histogram exhibits two local minima, at $X_{\min } \simeq 0.2$ and $X_{\max } \simeq 0.9$, which mark the scalar boundaries of the mixed-fluid region. Intermediate values exhibit two prominent compositions, corresponding to each of the (parts of the) large-scale structures captured (this scalar histogram derives from a single realization). A mean intermediate value of $X_{\text {mix }} \simeq 0.53$, between $X_{\min }$ and $X_{\max }$, can be calculated from the p.d.f. data and is indicated by the dashed-line vertical arrow in figure 19.

The resulting boundary level sets are depicted in figure 20. The scalar field in the mixed-fluid region can now be represented by a single composition. The scaled wavefront-phase function, computed using the model with a homogeneously mixed fluid within the turbulent region, i.e. setting $X(x, z)=X_{\text {mix }}$ in the mixed-fluid interboundary region within the bounding isoscalar contours, as depicted in figure 20 , is shown in figure 21. It is also compared with the previously computed wavefront-phase function, for the same data, using the full scalar field (cf. figure 7).

The agreement between the full-field computation and the homogeneously mixedfluid model calculation of the instantaneous wavefront-phase profile is very good and validates the hypothesis implemented in this model. Namely, the main beam- 


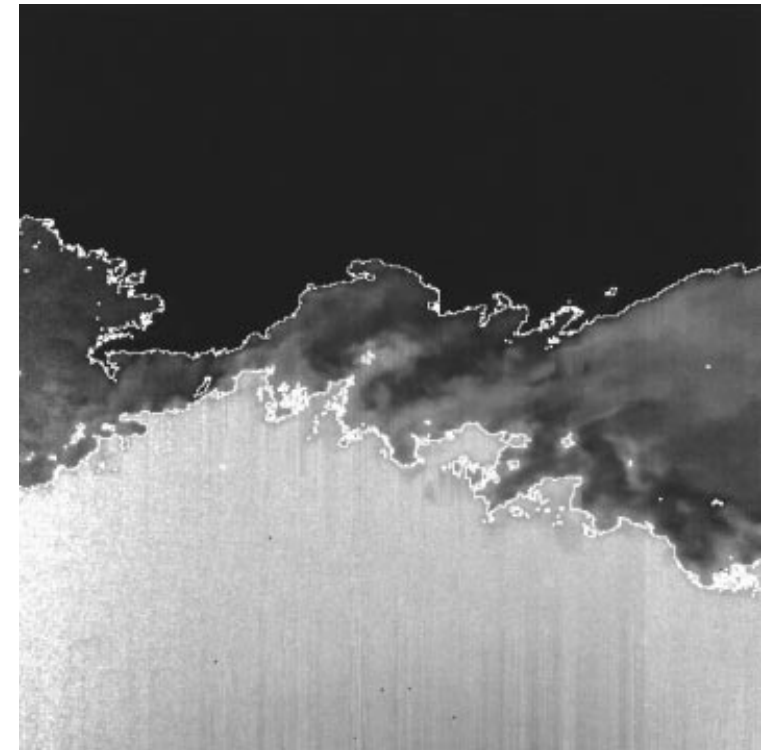

FiguRE 20. Boundary level sets for the subsonic, $M_{\mathrm{c}} \simeq 0.15$, scalar-field data in figure 4 .

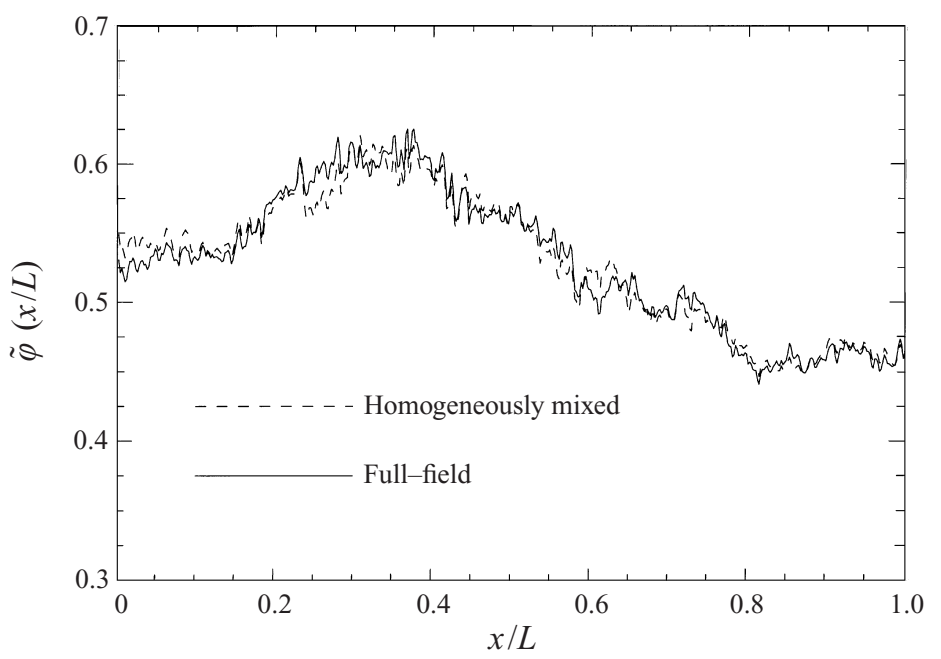

Figure 21. Comparison of shear-layer wavefront-phase functions. Dashed line: Homogeneously mixed, $X(x, z)=X_{\text {mix }}$, turbulent-region model. Solid line: Computed using the full scalar field (cf. figure 7).

propagation wavefront-phase contributions from high-Reynolds-number shear-layer flows stem from the complex geometry of relatively sharp interfaces between mixed (turbulent) and unmixed (free-stream) fluid, rather than the accumulated phase distortions from a statistically homogeneous variable index-of-refraction field.

Accepting this simple model at face value, we note that the reduction in the index-of-refraction-field information necessary, in the turbulent region, to describe the aero-optical interaction is substantial. For the aero-optical interaction of a laser sheet, the model effectively replaces the need for a full, two-dimensional scalar (molefraction) field, i.e. $X(x, z)$, with the specification of a (single) intermediate mole-fraction 
level, $X_{\text {mix }}$, within the (instantaneous) location of two isoscalar contours, on which $X(x, z)=X_{\min }$ and $X(x, z)=X_{\max }$. A similar reduction in the necessary information would be realized for a three-dimensional field, $X(x, y, z)$, as required to represent the propagation of a two-dimensional beam. In this case, the boundaries would be three-dimensional isoscalar surfaces, on which $X(x, y, z)=X_{\min }$ and $X(x, y, z)=X_{\max }$.

This simple model also facilitates the appreciation of the nature of the dominant aero-optical interaction, in terms of an effective variable-pitch variable-phase grating for a spatially developing shear layer and the optical-beam extent, i.e. whether its span is smaller than, comparable to, or larger than the local large-scale structures, as discussed in $\S 3$.

\section{Conclusions}

Investigations of flow structure and optical-beam propagation were conducted for two gas-phase flows: high-Reynolds-number $\left(0.2 \times 10^{6} \lesssim R e \lesssim 1.0 \times 10^{6}\right)$, variablecompressibility $\left(0.15 \lesssim M_{\mathrm{c}} \lesssim 0.96\right)$ shear layers, and moderate-Reynolds-number $\left(9 \times 10^{3} \lesssim R e \lesssim 30 \times 10^{3}\right)$ turbulent jets. These involved direct spatial measurements of the variable index-of-refraction field in the turbulent region. Measured scalarfield data permit wavefront-phase path integrals to be computed numerically along selected directions. These were chosen to be in the cross-stream direction in both flows and permitted the study of aero-optical interactions, within the geometricaloptics approximation. The resulting wavefront-phase functions indicate the dominant influence of the large-scale structure geometry and dynamics. Spatial wavefront-phase spectra indicate a weak sensitivity to compressibility for the turbulent shear layers and virtually no sensitivity to Reynolds number for turbulent jets. The latter is in accord with previous, gas-phase-jet scalar-field studies, but not with the behaviour documented for liquid-phase flows.

The ability of high-Re turbulence to homogenize the flow within effective separatrices in the convective large-scale-structure frame, directly confirmed in the present data, permits a simple model to be constructed for high-Re shear layers that is particularly successful in representing the aero-optical interaction in terms of the instantaneous wavefront-phase functions.

The aero-optical interactions for both flows may be described as producing phase gratings, whose qualitative effects depend on the transverse extent of the optical beam, as measured in terms of the local large-scale-structure sizes. For optical beams with extent smaller than or comparable to the large-scale-structure sizes, the effects may be described as those of local thin left/right wedges (prisms) and positive/negative lenses. Depending on the index-of-refraction gradient magnitudes, significant beamsteering, focusing (caustics), or defocusing may be expected to result in the optical far field from such aero-optical interactions. All three types of interactions remove optical-beam power from a receiving aperture in the far field.

This work was sponsored by AFOSR Grant F49620-94-1-0283 and completed under F49620-98-1-0052 and F49620-00-1-0036. We would like to acknowledge the expert assistance by Earl Dahl in the execution of both the shear-layer and jet experiments, Dan Lang for the design of the data-acquisition and computer-network systems that were essential for this work, the help of Pavel Svitek with some of the figures, Michael Slessor for his participation in the shear-layer experiments that generated the matching Schlieren images and his assistance with the text, and Ömer Savas for assistance with the text. 


\section{REFERENCES}

BAвсоCK, H. W. 1953 The possibility of compensating astronomical seeing. Publ. Astron. Soc. Pac. 65, 229-236.

BABCOCK, H. W. 1990 Adaptive optics revisited. Science 249, 253-257.

Born, M. \& Wolf, E. 1993 Principles of Optics, 6th edn. Pergamon.

Broadwell, J. E. \& Breidenthal, R. E. 1982 A simple model of mixing and chemical reaction in a turbulent shear layer. J. Fluid Mech. 125, 397-410.

Broadwell, J. E. \& Mungal, M. G. 1991 Large-scale structures and molecular mixing. Phys. Fluids A 3, 1193-1206.

Catrakis, H. J. \& Dimotakis, P. E. 1996 Mixing in turbulent jets: scalar measures and isosurface geometry. J. Fluid Mech. 317, 369-406.

Chernov, L. A. 1960 Wave Propagation in a Random Medium. McGraw-Hill.

CHOw, P. 1975 Perturbation methods in stochastic wave propagation. SIAM Rev. 17, 57-81.

Cicchiello, J. M. \& Jumper, E. J. 1997 Far-field optical degradation due to near-field transmission through a turbulent heated jet. Appl. Optics 36, 6441-6452.

Clemens, N. T. \& Mungal, M. G. 1992 Two- and three-dimensional effects in the supersonic mixing layer. AIAA J. 30, 973-981.

Clemens, N. T. \& Mungal, M. G. 1995 Large-scale structure and entrainment in the supersonic mixing layer. J. Fluid Mech. 284, 171-216.

Clifford, S. F. 1978 The classical theory of wave propagation in a turbulent medium. In Laser Beam Propagation in the Atmosphere (ed. J. W. Strohbehn). Springer.

Coons, S. A. 1967 Surfaces for computer-aided design of space forms. Massachusetts Institute of Technology, Rep. MAC-TR-41.

Dimotakis, P. E. 1986 Two-dimensional shear-layer entrainment. AIAA J. 24, 1791-1796.

Dimotakis, P. E. 1991a On the convection velocity of turbulent structures in supersonic shear layers. AIAA Paper 91-1724.

Diмотакis, P. E. $1991 b$ Turbulent free shear layer mixing and combustion. High Speed Flight Propulsion Systems, in Progress in Astronautics and Aeronautics, vol. 137, Ch. 5, pp. 265-340.

Dimotakis, P. E. 1993 Some issues on turbulent mixing and turbulence. GALCIT Rep. FM93-1a.

Dimotakis, P. E. 2000 The mixing transition in turbulence. J. Fluid Mech. 409, 69-97.

Dowling, D. R. \& Dimotakis, P. E. 1990 Similarity of the concentration field of gas-phase turbulent jets. J. Fluid Mech. 218, 109-141.

DYER, T. M. 1979 Rayleigh scattering measurements of time-resolved concentration in a turbulent propane jet. AIAA J. 17, 912-914.

Elliott, G. S., Samimy, M. \& Arnette, S. A. 1995 The characteristics and evolution of large-scale structures in compressible mixing layers. Phys. Fluids 7, 864-876.

FiEDLER, H. E. 1975 On turbulence structure and mixing mechanism in free turbulent shear flows. Turbulent Mixing in Non-Reactive and Reactive Flows, pp. 381-409. Plenum.

Fitzgerald, E. J. \& Jumper, E. J. 1998 Shear layer optical distortions due to compressibility vs. passive scalars. AIAA Paper 98-2834.

Fourguette, D. C., Bond, C. L. \& Dimotakis, P. E. 1993 Scalar field measurements in supersonic shear layers. Bull. Am. Phys. Soc. 38, 2202.

Fourguette, D. C., Dimotakis, P. E. \& Ching, W.-K. 1995 Whole-field index-of-refraction measurements in turbulent non-reacting jets. AIAA Paper 95-1980.

Fourguette, D., Mungal, M. G. \& Dibble, R. 1990 Time evolution of the shear layer of an axisymmetric supersonic jet at matched conditions. AIAA J. 29, 1123-1130.

Gilbert, K. G. $1982 a$ Overview of aero-optics. Progress in Astronautics and Aeronautics: AeroOptical Phenomena, vol. 80, pp. 1-9.

GILBERT, K. G. $1982 b$ Lear jet boundary-layer/shear-layer laser propagation measurements. Progress in Astronautics and Aeronautics: Aero-Optical Phenomena, vol. 80, 249-260.

GILBRECH, R. J. 1991 An experimental investigation of chemically-reacting, gas-phase turbulent jets. $\mathrm{PhD}$ thesis, California Institute of Technology.

Goodman, J. W. 1985 Statistical Optics. Wiley.

HALL, J. L. 1991 An experimental investigation of structure, mixing and combustion in compressible turbulent shear layers. PhD thesis, California Institute of Technology.

Hall, J. L., Dimotakis, P. E. \& Rosemann, H. 1993 Experiments in non-reacting compressible shear layers. AIAA J. 31, 2247-2254. 
Havener, G. 1992 Optical wave front variance: a study on analytical models in use today. AIAA Paper 92-0654.

JumPeR, E. J. \& Hugo, R. J. 1992 Optical phase distortion due to turbulent fluid density fields: Quantification using the small-aperture beam technique. AIAA Paper 92-3020.

JumPeR, E. J. \& HugO, R. J. 1995 Quantification of aero-optical phase distortion using the smallaperture beam technique. AIAA J. 33, 2151-2157.

Kelsall, D. 1973 Optical 'seeing' through the atmosphere by an interferometric technique. J. Opt. Soc. Am. 63, 1472-1484.

Kelsall, D. 1982 Optical measurements of degradation in aircraft boundary layers. Progress in Astronautics and Aeronautics: Aero-Optical Phenomena, vol. 80, pp. 261-293.

Klein, H. H., Malley, M. M., Sapp, O., Shough, D., Sutton, D., Sutton, G. W. \& Yu, J. H.-Y. 1990 Experimental measurements of the optical path difference of a four-meter dual aerocurtain. Propagation of High-Energy Laser Beams Through the Earth's Atmosphere, SPIE Proc. 1221.

KonRAD, J. H. 1976 An experimental investigation of mixing in two-dimensional turbulent shear flows with applications of diffusion-limited chemical reactions. PhD thesis, California Institute of Technology.

Liepmann, H. W. 1952 Deflection and diffusion of a light ray passing through a boundary layer. Douglas Aircraft Company Rep. SM-14397.

Malley, M. M., Sutton, G. W. \& Kincheloe, N. 1992 Beam-jitter measurements of turbulent aero-optical path differences. Appl. Optics 31, 4440-4443.

McMackin, L., Masson, B., Clark, N., Bishop, K., Pierson, R. \& Chen, E. 1995 Hartmann wave front sensor studies of dynamic organized structures in flowfields. AIAA J. 33, 2158-2164.

Miller, P. L. 1991 Mixing in high Schmidt number turbulent Jets. PhD thesis, California Institute of Technology.

Miller, P. L. \& Dimotakis, P. E. 1991 Reynolds number dependence of scalar fluctuations in a high Schmidt number turbulent jet. Phys. Fluids A 3, 1156-1163.

Miller, P. L. \& Dimotakis, P. E. 1996 Measurements of scalar power spectra in high Schmidt number turbulent jets. J. Fluid Mech. 308, 129-146.

Papamoschou, D. 1989 Structure of the compressible turbulent shear layer. AIAA Paper 89-0126.

PAPAMOSCHOU, D. 1991 Structure of the compressible turbulent shear layer. AIAA J. 29, 680-681.

PARmentier, E. M. \& GreenberG, R. A. 1973 Supersonic flow aerodynamic windows for high-power laser. AIAA J. 11, 943-949.

Penney, C. M. 1969 Light scattering in terms of oscillator strengths and refractive indices. J. Opt. Soc. Am. 59, 34-42.

RodDier, F. 1981 The effects of atmospheric turbulence in optical astronomy. Progress in Optics, vol. XIX (ed. E. Wolf). North-Holland.

Rosemann, H., Dimotakis, P. E. \& Hall, J. 1992 Flow visualization in compressible turbulent shear layers. IUTAM Symp., Eddy Structure Identification in Free Turbulent Shear Flows, 12 14 October 1992, Poitiers, France.

Sandham, N. D. \& ReYnolds, W. C. 1991 Three-dimensional simulations of large eddies in the compressible mixing layer. J. Fluid Mech. 224, 133-158.

Slessor, M. D. 1998 Aspects of turbulent-shear-layer dynamics and mixing. PhD thesis, California Institute of Technology.

Slessor, M. D., Bond, C. L. \& Dimotakis, P. E. 1998 Turbulent shear-layer mixing at high Reynolds numbers: effects of inflow conditions. J. Fluid Mech. 376, 115-138.

SteinmetZ, W. J. 1982 Second moments of optical degradation due to a thin turbulent layer. Progress in Astronautics and Aeronautics: Aero-Optical Phenomena, vol. 80, pp. 78-100.

Sutton, G. W. 1969 Effect of turbulent fluctuations in an optically active medium. AIAA J. 7, 1737-1743.

Sutton, G. W. 1985 Aero-optical foundations and applications. AIAA J. 23, 1525-1537.

TatarskiI, V. I. 1961 The Effects of the Turbulent Atmosphere on Wave Propagation. McGraw-Hill.

Truman, C. R. \& LeE, M. J. 1990 Effects of organized turbulence structures on the phase distortion in a coherent optical beam propagating through a turbulent shear flow. Phys. Fluids A 2, 851-857.

Tyson, R. K. 1991 Principles of Adaptive Optics. Academic.

Wissler, J. B. \& Roshko, A. 1992 Transmission of thin light beams through turbulent mixing layers. AIAA Paper 92-0658.

Yip, B., Lyons, M., Long, M., Mungal, M. G., Barlow, R. \& Dibble, R. 1989 Visualization of a supersonic underexpanded jet by planar Rayleigh scattering. Gallery of fluid motion. Phys. Fluids A 1, 1449. 